\title{
Competition and duality correspondence between inhomogeneous fermion-antifermion and fermion-fermion condensations in the $\mathrm{NJL}_{2}$ model
}

\author{
D. Ebert ${ }^{1)}$, T.G. Khunjua ${ }^{2)}$, K.G. Klimenko ${ }^{3,4)}$, and V.Ch. Zhukovsky ${ }^{2)}$ \\ 1) Institute of Physics, Humboldt-University Berlin, 12489 Berlin, Germany \\ 2) Faculty of Physics, Moscow State University, 119991, Moscow, Russia \\ 3) Institute for High Energy Physics, 142281, Protvino, Moscow Region, Russia and \\ 4) Dubna International University (Protvino branch), 142281, Protvino, Moscow Region, Russia
}

\begin{abstract}
We investigate the possibility of spatially homogeneous and inhomogeneous chiral fermionantifermion condensation and superconducting fermion-fermion pairing in the $(1+1)$-dimensional model by Chodos et al. [ Phys. Rev. D 61, 045011 (2000)] generalized to continuous chiral invariance. The consideration is performed at nonzero values of temperature $T$, electric charge chemical potential $\mu$ and chiral charge chemical potential $\mu_{5}$. It is shown that at $G_{1}<G_{2}$, where $G_{1}$ and $G_{2}$ are the coupling constants in the fermion-antifermion and fermion-fermion channels, the $\left(\mu, \mu_{5}\right)$-phase structure of the model is in a one-to-one correspondence with the phase structure at $G_{1}>G_{2}$ (called duality correspondence). Under the duality transformation the (inhomogeneous) chiral symmetry breaking (CSB) phase is mapped into the (inhomogeneous) superconducting (SC) phase and vice versa. If $G_{1}=G_{2}$, then the phase structure of the model is self-dual. Nevertheless, the degeneracy between the CSB and SC phases is possible in this case only when there is a spatial inhomogeneity of condensates.
\end{abstract}

\section{INTRODUCTION}

In recent years much attention has been devoted to the investigation of dense quark (or baryonic) matter. The interest is motivated by the possible existence of quark matter inside compact stars or its creation in heavy ion collisions. In many cases, as e.g. in the above-mentioned heavy ion collision experiments the quark matter densities are not too high, so the consideration of its properties is not possible in the framework of perturbative weak coupling QCD. Usually, different effective theories such as the Nambu - Jona-Lasinio (NJL) model, $\sigma$ model etc. are more adequate in order to study the QCD and quark matter phase diagram in this case. A variety of spatially nonuniform (inhomogeneous) quark matter phases related to chiral symmetry breaking, color superconductivity, and charged pion condensation phenomenon etc. (see, e.g., [1-12] and references therein) was predicted in the framework of NJL-like models at rather low values of temperature and baryon density. (A recent interesting review on current model results for inhomogeneous phases in $(3+1)$-dimensional systems is presented in [13].)

Moreover, the phenomenon of spatially nonuniform quark pairing was also intensively investigated within different $(1+1)$-dimensional models which can mimic qualitatively the QCD phase diagram. In this connection, it is necessary to mention Gross-Neveu (GN) type models with four-fermion interactions, symmetrical with respect to the discrete or continuous chiral transformations (in the last case we shall use for such models the notation $\mathrm{NJL}_{2}$ ) and extended by baryon and isospin chemical potentials. In the framework of these models both the inhomogeneous chiral [1618] and charged pion condensation phenomena were considered [19, 20]. (In order to overcome the prohibition on the spontaneous breaking of a continuous symmetry in $(1+1)$ dimensions, the consideration is usually performed in the limit of large $N$, where $N$ is the number of quark multiplets.) Inhomogeneous phases in some one-dimensional organic materials and nonrelativistic Fermi gases were recently studied, correspondingly, in [14] and [15] in terms of $(1+1)$-dimensional theories with four-fermion interaction.

Among a variety of GN-type models, there is one which describes competition between quark-antiquark (or chiral) and quark-quark (or superconducting) pairing at nonzero temperature $T$ and quark number chemical potential $\mu$ [21]. Originally, the model was called for to shed new light on the color superconductivity phenomenon in real dense quark matter. Moreover, in [21] the consideration is performed in the supposition that chiral and superconducting condensates are spatially homogeneous. In this case it was shown there that if $G_{1}>G_{2}$, where $G_{1}$ and $G_{2}$ are the coupling constants in the chiral and superconducting channels, correspondingly, then at rather high values of quark number chemical potential $\mu$ the superconducting phase is realized in the system.

Since in the true ground state of any system with nonzero density the condensates could be inhomogeneous, the aim of the present paper is to investigate such a possibility. Namely, we shall study the phase structure of the extended model [21] (which is symmetric with respect to continuous $U_{A}(1)$ chiral group), assuming that both quark-antiquark and quark-quark condensates might have a spatial inhomogeneity in the form of the Fulde-Ferrel single plane wave ansatz [22], for simplicity. Moreover, in addition to the particle (or quark) number chemical potential $\mu$, we also introduce into consideration the chiral charge chemical potential $\mu_{5}$, which is responsible for a nonzero chiral charge density $n_{5}$, i.e. to a nonzero imbalance between densities of left- and right-handed quarks (fermions). In literature, there are some investigations of QCD-like effective theories with $\mu$ and $\mu_{5}$ chemical potentials, related to a possible parity breaking phenomena of dense quark gluon plasma (see, e.g., [23, 24]). Moreover, it was recently established that in heavy ion collision experiments a nonzero chiral charge density $n_{5}$ can be induced, leading to the so-called chiral magnetic effect [25, 26]. So, we hope that studying the above- mentioned (1+1)-dimensional NJL model with 
two chemical potentials, $\mu$ and $\mu_{5}$, one can shed new light on the new phenomena of the dense baryonic matter.

The paper is organized as follows. In Sec. II the duality property of the model is established. It means that there is a correspondence between properties (phase structure) of the model at $G_{1}<G_{2}$ and $G_{1}>G_{2}$. After obtaining the thermodynamic potential (TDP), we will first investigate it in the next Sec. III under the supposition that both superconducting and chiral condensates are spatially homogeneous. In this section a rather rich $\left(\mu, \mu_{5}\right)$-phase structure of the model is established at $G_{1}>G_{2}$. In addition, we will show here that there is an invariance of the TDP with respect to a duality transformation (when $G_{1} \leftrightarrow G_{2}, \mu \leftrightarrow \mu_{5}$ and superconductivity $\leftrightarrow$ chiral symmetry breaking). As a result, the $\left(\mu, \mu_{5}\right)$-phase structure of the model at $G_{1}<G_{2}$ is a dual mapping of the phase portrait at $G_{1}>G_{2}$. In Sec. IV the phase structure of the model is investigated in the assumption that both condensates might be spatially inhomogeneous. Then at $G_{1}>G_{2}$ the chiral density wave phase is realized for arbitrary values of $\mu \neq 0$ and $\mu_{5} \neq 0$. On the other hand, at $G_{1}<G_{2}$ there is an inhomogeneous superconducting phase in the whole $\left(\mu, \mu_{5}\right)$ plane. Note, that there is a dual correspondence between these phases. Finally, Sec. V presents a summary and some concluding remarks. The discussion of some technical problems are relegated to four Appendixes.

\section{THE MODEL AND ITS THERMODYNAMIC POTENTIAL}

\section{A. The duality property of the model}

Our investigation is based on a (1+1)-dimensional NJL-type model with massless fermions belonging to a fundamental multiplet of the $O(N)$ flavor group. Its Lagrangian describes the interaction in the fermion-antifermion and scalar fermion-fermion channels,

$$
\begin{array}{r}
L=\sum_{k=1}^{N} \bar{\psi}_{k}\left[\gamma^{\nu} i \partial_{\nu}+\mu \gamma^{0}+\mu_{5} \gamma^{0} \gamma^{5}\right] \psi_{k}+\frac{G_{1}}{N}\left[\left(\sum_{k=1}^{N} \bar{\psi}_{k} \psi_{k}\right)^{2}+\left(\sum_{k=1}^{N} \bar{\psi}_{k} i \gamma^{5} \psi_{k}\right)^{2}\right] \\
+\frac{G_{2}}{N}\left(\sum_{k=1}^{N} \psi_{k}^{T} \epsilon \psi_{k}\right)\left(\sum_{j=1}^{N} \bar{\psi}_{j} \epsilon \bar{\psi}_{j}^{T}\right)
\end{array}
$$

where $\mu$ is a fermion number chemical potential (conjugated to a fermion, or electric charge, number density) and $\mu_{5}$ is an axial chemical potential conjugated to a nonzero density of chiral charge $n_{5}=n_{R}-n_{L}$, which represents an imbalance in densities of the right- and left-handed fermions [25]. As it is noted above, all fermion fields $\psi_{k}$ $(k=1, \ldots, N)$ form a fundamental multiplet of the $O(N)$ group. Moreover, each field $\psi_{k}$ is a two-component Dirac spinor (the symbol $T$ denotes the transposition operation). The quantities $\gamma^{\nu}(\nu=0,1), \gamma^{5}$, and $\epsilon$ in (1) are matrices in the two-dimensional spinor space,

$$
\gamma^{0}=\left(\begin{array}{cc}
0 & 1 \\
1 & 0
\end{array}\right) ; \quad \gamma^{1}=\left(\begin{array}{cc}
0 & -1 \\
1 & 0
\end{array}\right) \equiv-\epsilon ; \quad \gamma^{5}=\gamma^{0} \gamma^{1}=\left(\begin{array}{cc}
1 & 0 \\
0 & -1
\end{array}\right)
$$

It follows from (2) that $\mu_{5} \gamma^{0} \gamma^{5}=\mu_{5} \gamma^{1}$. Clearly, the Lagrangian $L$ is invariant under transformations from the internal $O(N)$ group, which is introduced here in order to make it possible to perform all the calculations in the framework of the nonperturbative large- $N$ expansion method. Physically more interesting is that the model (1) is invariant under transformations from the $U_{V}(1) \times U_{A}(1)$ group, where $U_{V}(1)$ is the fermion number conservation group, $\psi_{k} \rightarrow \exp (i \alpha) \psi_{k}(k=1, \ldots, N)$, and $U_{A}(1)$ is the group of continuous chiral transformations, $\psi_{k} \rightarrow \exp \left(i \alpha^{\prime} \gamma^{5}\right) \psi_{k}$ $(k=1, \ldots, N)$. 1 The linearized version of Lagrangian (1) that contains auxiliary scalar bosonic fields $\sigma(x), \pi(x)$, $\Delta(x), \Delta^{*}(x)$ has the following form:

$$
\begin{aligned}
\mathcal{L} \equiv \mathcal{L}\left(G_{1}, G_{2} ; \mu, \mu_{5}\right) & =\bar{\psi}_{k}\left[\gamma^{\nu} i \partial_{\nu}+\mu \gamma^{0}+\mu_{5} \gamma^{1}-\sigma-i \gamma^{5} \pi\right] \psi_{k} \\
& -\frac{N}{4 G_{1}}\left(\sigma^{2}+\pi^{2}\right)-\frac{N}{4 G_{2}} \Delta^{*} \Delta-\frac{\Delta^{*}}{2}\left[\psi_{k}^{T} \epsilon \psi_{k}\right]-\frac{\Delta}{2}\left[\bar{\psi}_{k} \epsilon \bar{\psi}_{k}^{T}\right]
\end{aligned}
$$

(Here and in what follows, summation over repeated indices $k=1, \ldots, N$ is implied.) Clearly, the Lagrangians (1) and (3) are equivalent, as can be seen by using the Euler-Lagrange equations of motion for scalar bosonic fields which take the form

$$
\sigma(x)=-2 \frac{G_{1}}{N}\left(\bar{\psi}_{k} \psi_{k}\right), \quad \pi(x)=-2 \frac{G_{1}}{N}\left(\bar{\psi}_{k} i \gamma^{5} \psi_{k}\right), \quad \Delta(x)=-2 \frac{G_{2}}{N}\left(\psi_{k}^{T} \epsilon \psi_{k}\right), \quad \Delta^{*}(x)=-2 \frac{G_{2}}{N}\left(\bar{\psi}_{k} \epsilon \bar{\psi}_{k}^{T}\right) .
$$

\footnotetext{
${ }^{1}$ Earlier in [21] a similar model symmetric under discrete $\gamma^{5}$ chiral transformation was investigated. However, only the possibility for the spatially homogeneous chiral and difermion condensates was considered there. In our paper, the invariance of the model considered by Chodos et al. 21] is generalized to the case of continuous chiral symmetry in order to study the inhomogeneous chiral condensates in the form of chiral spirals (or chiral density waves).
} 
One can easily see from (44) that the (neutral) fields $\sigma(x)$ and $\pi(x)$ are real quantities, i.e. $(\sigma(x))^{\dagger}=\sigma(x),(\pi(x))^{\dagger}=$ $\pi(x)$ (the superscript symbol $\dagger$ denotes the Hermitian conjugation), but the (charged) difermion scalar fields $\Delta(x)$ and $\Delta^{*}(x)$ are Hermitian conjugated complex quantities, so $(\Delta(x))^{\dagger}=\Delta^{*}(x)$ and vice versa. Clearly, all the fields (4) are singlets with respect to the $O(N)$ group. 2 If the scalar difermion field $\Delta(x)$ has a nonzero ground state expectation value, i.e. $\langle\Delta(x)\rangle \neq 0$, the Abelian fermion number $U_{V}(1)$ symmetry of the model is spontaneously broken down. However, if $\langle\sigma(x)\rangle \neq 0$ then the continuous chiral symmetry of the model is spontaneously broken.

Before studying the thermodynamics of the model, we want first of all to consider its duality property. To this end, it is very useful to form an infinite set $\mathcal{F}$ composed of all Lagrangians $\mathcal{L}\left(G_{1}, G_{2} ; \mu, \mu_{5}\right)$ (3) when the free model parameters $G_{1}, G_{2}, \mu$ and $\mu_{5}$ take arbitrary admissible values, i.e. $\mathcal{L}\left(G_{1}, G_{2} ; \mu, \mu_{5}\right) \in \mathcal{F}$ at arbitrary fixed values of coupling constants $G_{1}>0, G_{2}>0$ and chemical potentials $\mu, \mu_{5}$. Then, let us perform in (3) the so-called PauliGursey transformation of spinor fields [27], accompanied with corresponding simultaneous transformations of auxiliary scalar fields (4),

$$
\psi_{k}(x) \longrightarrow \frac{1}{2}\left(1-\gamma^{5}\right) \psi_{k}(x)+\frac{1}{2}\left(1+\gamma^{5}\right) \epsilon \bar{\psi}_{k}^{T}(x) ; \quad \sigma(x) \rightleftarrows \frac{\Delta(x)+\Delta^{*}(x)}{2} ; \quad \pi(x) \rightleftarrows \frac{\Delta(x)-\Delta^{*}(x)}{2 i} .
$$

Taking into account that all spinor fields anticommute with each other, it is easy to see that under the action of the transformations (5) each element (auxiliary Lagrangian) $\mathcal{L}\left(G_{1}, G_{2} ; \mu, \mu_{5}\right)$ of the set $\mathcal{F}$ is transformed into another element of the set $\mathcal{F}$ according to the following rule

$$
\mathcal{L}\left(G_{1}, G_{2} ; \mu, \mu_{5}\right) \longrightarrow \mathcal{L}\left(G_{2}, G_{1} ;-\mu_{5},-\mu\right) \in \mathcal{F},
$$

i.e. the set $\mathcal{F}$ is invariant under the field transformations (5). Owing to the relation (6) there is a connection between properties of the model when free model parameters $G_{1}, G_{2}, \mu$ and $\mu_{5}$ vary in different regions. Due to this reason, we will call the relation (6) the duality property of the model.

\section{B. The thermodynamic potential at $T=0$}

We begin an investigation of a phase structure of the four-fermion model (1) using the equivalent semibosonized Lagrangian (3). In the leading order of the large- $N$ approximation, the effective action $\mathcal{S}_{\text {eff }}\left(\sigma, \pi, \Delta, \Delta^{*}\right)$ of the considered model is expressed by means of the path integral over fermion fields:

$$
\exp \left(i \mathcal{S}_{\mathrm{eff}}\left(\sigma, \pi, \Delta, \Delta^{*}\right)\right)=\int \prod_{l=1}^{N}\left[d \bar{\psi}_{l}\right]\left[d \psi_{l}\right] \exp \left(i \int \mathcal{L} d^{2} x\right)
$$

where

$$
\mathcal{S}_{\text {eff }}\left(\sigma, \pi, \Delta, \Delta^{*}\right)=-\int d^{2} x\left[\frac{N}{4 G_{1}}\left(\sigma^{2}(x)+\pi^{2}(x)\right)+\frac{N}{4 G_{2}} \Delta(x) \Delta^{*}(x)\right]+\widetilde{\mathcal{S}}_{\text {eff }}
$$

The fermion contribution to the effective action, i.e. the term $\widetilde{\mathcal{S}}_{\text {eff }}$ in (7), is given by

$$
\exp \left(i \widetilde{\mathcal{S}}_{\mathrm{eff}}\right)=\int \prod_{l=1}^{N}\left[d \bar{\psi}_{l}\right]\left[d \psi_{l}\right] \exp \left\{i \int\left[\bar{\psi}_{k}\left(\gamma^{\nu} i \partial_{\nu}+\mu \gamma^{0}+\mu_{5} \gamma^{1}-\sigma-i \gamma^{5} \pi\right) \psi_{k}-\frac{\Delta^{*}}{2}\left(\psi_{k}^{T} \epsilon \psi_{k}\right)-\frac{\Delta}{2}\left(\bar{\psi}_{k} \epsilon \bar{\psi}_{k}^{T}\right)\right] d^{2} x\right\} .
$$

The ground state expectation values $\langle\sigma(x)\rangle,\langle\pi(x)\rangle$, etc. of the composite bosonic fields are determined by the saddle point equations,

$$
\frac{\delta \mathcal{S}_{\mathrm{eff}}}{\delta \sigma(x)}=0, \quad \frac{\delta \mathcal{S}_{\mathrm{eff}}}{\delta \pi(x)}=0, \quad \frac{\delta \mathcal{S}_{\mathrm{eff}}}{\delta \Delta(x)}=0, \quad \frac{\delta \mathcal{S}_{\mathrm{eff}}}{\delta \Delta^{*}(x)}=0
$$

In vacuum, i.e. in the state corresponding to an empty space with zero particle density and zero values of the chemical potentials $\mu$ and $\mu_{5}$, the above-mentioned quantities $\langle\sigma(x)\rangle$, etc. do not depend on space coordinates. However, in a dense medium, when $\mu \neq 0$ and/or $\mu_{5} \neq 0$, the ground state expectation values of bosonic fields (4) might have a nontrivial dependence on the spatial coordinate $x$. In particular, in this paper we will use the following ansatz:

$$
\langle\sigma(x)\rangle=M \cos (2 b x), \quad\langle\pi(x)\rangle=M \sin (2 b x), \quad\langle\Delta(x)\rangle=\Delta \exp \left(2 i b^{\prime} x\right), \quad\left\langle\Delta^{*}(x)\right\rangle=\Delta \exp \left(-2 i b^{\prime} x\right),
$$

where $M, b, b^{\prime}$ and $\Delta$ are real constant quantities. (It means that we suppose for $\langle\sigma(x)\rangle$ and $\langle\pi(x)\rangle$ the chiral spiral (or chiral density wave) ansatz, and the Fulde-Ferrel [22] single plane wave ansatz for difermion condensates.) In fact,

\footnotetext{
${ }^{2}$ Note that the $\Delta(x)$ field is a flavor $O(N)$ singlet, since the representations of this group are real.
} 
they are coordinates of the global minimum point of the thermodynamic potential $\Omega\left(M, b, b^{\prime}, \Delta\right)$. 3 In the leading order of the large $N$-expansion it is defined by the following expression:

$$
\int d^{2} x \Omega\left(M, b, b^{\prime}, \Delta\right)=-\left.\frac{1}{N} \mathcal{S}_{\text {eff }}\left\{\sigma(x), \pi(x), \Delta(x), \Delta^{*}(x)\right\}\right|_{\sigma(x)=\langle\sigma(x)\rangle, \pi(x)=\langle\pi(x)\rangle, \ldots},
$$

which gives

$$
\begin{aligned}
\int d^{2} x \Omega\left(M, b, b^{\prime}, \Delta\right)= & \int d^{2} x\left(\frac{M^{2}}{4 G_{1}}+\frac{\Delta^{2}}{4 G_{2}}\right)+\frac{i}{N} \ln \left(\int \prod _ { l = 1 } ^ { N } [ d \overline { \psi } _ { l } ] [ d \psi _ { l } ] \operatorname { e x p } \left(i \int d ^ { 2 } x \left[\bar{\psi}_{k} \mathcal{D} \psi_{k}\right.\right.\right. \\
& \left.\left.\left.-\frac{\Delta \exp \left(-2 i b^{\prime} x\right)}{2}\left(\psi_{k}^{T} \epsilon \psi_{k}\right)-\frac{\Delta \exp \left(2 i b^{\prime} x\right)}{2}\left(\bar{\psi}_{k} \epsilon \bar{\psi}_{k}^{T}\right)\right]\right)\right),
\end{aligned}
$$

where $\mathcal{D}=\gamma^{\rho} i \partial_{\rho}+\mu \gamma^{0}+\mu_{5} \gamma^{1}-M \exp \left(2 i \gamma^{5} b x\right)$. In principle, one way to evaluate the path integral in (11) is to extend the technique of the paper [18], where a more simple model with single quark-antiquark channel of interaction was investigated, to the case under consideration, i.e. to the GN model (1) with additional superconducting interaction of quarks. The rigorous method of [18] is based on finding the resolvent function corresponding to the Hamiltonian of the system. However, technically it is very difficult to use this approach in the framework of the model (1). So, in order to simplify the problem we first perform in (11) Weinberg (or chiral) transformation of spinor fields [28], $q_{k}=\exp \left[i\left(\gamma^{5} b-b^{\prime}\right) x\right] \psi_{k}$ and $\bar{q}_{k}=\bar{\psi}_{k} \exp \left[i\left(\gamma^{5} b+b^{\prime}\right) x\right]$. Since Weinberg transformation of fermion fields does not change the path integral measure in (11), 4 we see that the system is reduced by the Weinberg transformation from a spatially modulated to a uniform one; i.e. we obtain the following expression for the thermodynamic potential:

$$
\begin{aligned}
\int d^{2} x \Omega\left(M, b, b^{\prime}, \Delta\right) & =\int d^{2} x\left(\frac{M^{2}}{4 G_{1}}+\frac{\Delta^{2}}{4 G_{2}}\right) \\
& +\frac{i}{N} \ln \left(\int \prod_{l=1}^{N}\left[d \bar{q}_{l}\right]\left[d q_{l}\right] \exp \left(i \int d^{2} x\left[\bar{q}_{k} D q_{k}-\frac{\Delta}{2}\left(q_{k}^{T} \epsilon q_{k}\right)-\frac{\Delta}{2}\left(\bar{q}_{k} \epsilon \bar{q}_{k}^{T}\right)\right]\right)\right),
\end{aligned}
$$

where

$$
D=\gamma^{\nu} i \partial_{\nu}+(\mu-b) \gamma^{0}-M+\gamma^{1}\left(\mu_{5}-b^{\prime}\right) .
$$

The path integration in the expression (12) is evaluated in Appendix $\mathrm{A}$ (5 (see also [30] for similar integrals), so we have for the TDP

$$
\Omega\left(M, b, b^{\prime}, \Delta\right) \equiv \Omega^{u n}\left(M, b, b^{\prime}, \Delta\right)=\frac{M^{2}}{4 G_{1}}+\frac{\Delta^{2}}{4 G_{2}}+\frac{i}{2} \int \frac{d^{2} p}{(2 \pi)^{2}} \ln \left[\lambda_{1}(p) \lambda_{2}(p)\right],
$$

where $\lambda_{1,2}(p)$ are presented in (A8) and superscript "un" denotes the unrenormalized quantity. Note, the TDP (14) describes thermodynamics of the model at zero temperature $T$. In the following we will study the behavior of the global minimum point of this TDP as a function of dynamical variables $M, b, b^{\prime}, \Delta$ vs the external parameters $\mu$ and $\mu_{5}$ in two qualitatively different cases: (i) the case of homogeneous condensates, i.e. when in (10) and (14) both $b$ and $b^{\prime}$ are supposed from the very beginning, without any proof, to be zero, and (ii) the case of spatially inhomogeneous condensates, i.e. when the quantities $b$ and $b^{\prime}$ are defined dynamically by the gap equations of the TDP (14). Moreover, the influence of temperature $T$ on the phase structure is also taken into account.

\section{THE HOMOGENEOUS CASE OF THE ANSATZ (10) FOR CONDENSATES: $b=0$ AND $b^{\prime}=0$}

\section{A. Dual invariance of the TDP}

In the present section we suppose that all the condensates are spatially homogeneous, i.e. we put in the ansatz (10) and in the TDP (14) $b \equiv 0$ and $b^{\prime} \equiv 0$. So, the TDP is considered $a$ priori as a function of only two variables, $M$ and $\Delta$ ( $\mu$ and $\mu_{5}$ are treated as external parameters). Note that the subject and results of the section are largely

\footnotetext{
${ }^{3}$ Here and in what follows we will use a conventional notation "global" minimum in the sense that among all our numerically found local minima the thermodynamical potential takes in their case the lowest value. This does not exclude the possibility that there exist other inhomogeneous condensates, different from (10), which lead to ground states with even lower values of the TDP.

${ }^{4}$ Strictly speaking, performing Weinberg transformation of fermion fields in (11), one can obtain in the path integral measure a factor, which however does not depend on the dynamical variables $M, \Delta, b$, and $b^{\prime}$. Hence, we ignore this unessential factor in the following calculations. Note that only in the case when there is an interaction between spinor and gauge fields there might appear a nontrivial, i.e. dependent on dynamical variables, path integral measure, generated by Weinberg transformation of spinors. This unobvious fact follows from the investigations by Fujikawa [29].

${ }^{5}$ In Appendix $\mathrm{A}$ we consider for simplicity the case $N=1$; however the procedure is easily generalized to the case with $N>1$.
} 
preparatory for considering the main purpose of the paper, i.e. to clarify (see the next section) a genuine ground state structure of the model in the framework of the inhomogeneous ansatz (10) for condensates.

Taking into account the expressions (A8) for $\lambda_{1,2}(p)$, we obtain the unrenormalized TDP in this case:

$$
\Omega^{u n}(M, \Delta)=\frac{M^{2}}{4 G_{1}}+\frac{\Delta^{2}}{4 G_{2}}+\frac{i}{2} \int \frac{d^{2} p}{(2 \pi)^{2}} \ln [\operatorname{det} \bar{B}(p)]
$$

where

$$
\begin{aligned}
\operatorname{det} \bar{B}(p)=\left.\lambda_{1}(p) \lambda_{2}(p)\right|_{b=0, b^{\prime}=0} & =\Delta^{4}-2 \Delta^{2}\left(p_{0}^{2}-p_{1}^{2}+M^{2}+\mu_{5}^{2}-\mu^{2}\right) \\
& +\left(M^{2}+\left(p_{1}-\mu_{5}\right)^{2}-\left(p_{0}-\mu\right)^{2}\right)\left(M^{2}+\left(p_{1}+\mu_{5}\right)^{2}-\left(p_{0}+\mu\right)^{2}\right) .
\end{aligned}
$$

Expanding the right-hand side of (16) in powers of $M$, one can obtain an equivalent expression for det $\bar{B}(p)$. Namely,

$$
\begin{aligned}
\operatorname{det} \bar{B}(p)=M^{4}-2 M^{2}\left(p_{0}^{2}-p_{1}^{2}\right. & \left.+\Delta^{2}+\mu^{2}-\mu_{5}^{2}\right) \\
& +\left(\Delta^{2}+\left(p_{1}-\mu\right)^{2}-\left(p_{0}-\mu_{5}\right)^{2}\right)\left(\Delta^{2}+\left(p_{1}+\mu\right)^{2}-\left(p_{0}+\mu_{5}\right)^{2}\right) .
\end{aligned}
$$

We would like to stress once more that there is an identical equality between the expressions (16) and (17).

Obviously, the function $\Omega^{u n}(M, \Delta)(15)$ is symmetric with respect to the transformations $M \rightarrow-M$ and/or $\Delta \rightarrow-\Delta$. Moreover, it is invariant under the transformations $\mu_{5} \rightarrow-\mu_{5}$ and/or $\mu \rightarrow-\mu$. 6 Hence, without loss of generality, we restrict ourselves by the constraints: $M \geq 0, \Delta \geq 0, \mu \geq 0$, and $\mu_{5} \geq 0$. However, there is one more discrete transformation of the TDP (15), which leaves it invariant. It follows from a comparison between (16) and (17). Indeed, if in (16) for $\operatorname{det} \bar{B}(p)$ the transformations $\mu \leftrightarrow \mu_{5}$ and $M \leftrightarrow \Delta$ are performed simultaneously, then the expression (17) will be obtained, which is equal to the original expression (16) for $\operatorname{det} \bar{B}(p)$. So the TDP (15) is invariant with respect to the following duality transformation $D$ :

$$
D: \quad G_{1} \longleftrightarrow G_{2}, \quad \mu \longleftrightarrow \mu_{5}, \quad M \longleftrightarrow \Delta
$$

Taking into account that the TDP (15) is symmetric with respect to $\mu_{5} \rightarrow-\mu_{5}$ and/or $\mu \rightarrow-\mu$, it is possible to conclude that the dual invariance $D$ of the TDP (15) is a particular realization of the dual property (6) of the initial model. Suppose now that at some fixed particular values of the model parameters, i.e. at $G_{1}=A, G_{2}=B$ and $\mu=\alpha$, $\mu_{5}=\beta$, the global minimum point of the TDP lies at the point $\left(M=M_{0}, \Delta=\Delta_{0}\right)$. Then it follows from the dual invariance $D(18)$ of the TDP that the permutation of the coupling constant and chemical potential values (i.e. at $G_{1}=B, G_{2}=A$ and $\left.\mu=\beta, \mu_{5}=\alpha\right)$ moves the global minimum point of the TDP to the point $\left(M_{=}=\Delta_{0}, \Delta=M_{0}\right)$. In particular, if in the original model with $G_{1}=A, G_{2}=0$ and $\mu=\alpha, \mu_{5}=0$ the global minimum point of the TDP lies at the point $\left(M=M_{0}, \Delta=0\right.$ ) (as a result, in this case the continuous chiral symmetry $U_{A}(1)$ is spontaneously broken down), then in the model with $G_{1}=0, G_{2}=A$ and $\mu=0, \mu_{5}=\alpha$ the global minimum point of the TDP lies at the point $\left(M=0, \Delta=M_{0}\right)$ and the symmetry $U_{V}(1)$ is spontaneously broken. The duality correspondence between these two particular cases of the original model (1) was discussed in [31]. (Even earlier, a special case with $\mu=\mu_{5}=0$ of the duality between chiral symmetry breaking and superconductivity phenomena was considered in the framework of the simplest two-dimensional Gross-Neveu model [32, 33].) Hence, a knowledge of a phase structure of the model (1) at $G_{1}<G_{2}$ is sufficient to construct, by applying the duality transformation $D$ (18), the phase structure at $G_{1}>G_{2}$; i.e. in the model under consideration there is a duality correspondence between chiral symmetry breaking and superconducting phases.

To investigate the TDP (15) it is necessary to renormalize it.

\section{B. The vacuum case: $\mu=0, \mu_{5}=0$}

First of all we will consider the renormalization procedure and the phase structure of the model in the vacuum case, i.e. when $\mu=0, \mu_{5}=0$. Putting $\mu=0$ and $\mu_{5}=0$ in (15), we have in this case the following expression for the unrenormalized effective potential $V_{0}^{u n}(M, \Delta)$ (in vacuum TDP is usually called an effective potential):

$$
V_{0}^{u n}(M, \Delta)=\frac{M^{2}}{4 G_{1}}+\frac{\Delta^{2}}{4 G_{2}}+\frac{i}{2} \int \frac{d^{2} p}{(2 \pi)^{2}} \ln \left[\left(p_{0}^{2}-p_{1}^{2}-(\Delta-M)^{2}\right)\left(p_{0}^{2}-p_{1}^{2}-(\Delta+M)^{2}\right)\right],
$$

Integrating in (19) over $p_{0}$ (see Appendix B in [20] for similar integrals) and cutting the integration $p_{1}$ region, $\left|p_{1}\right|<\Lambda$, one obtains the regularized effective potential $V_{0}^{r e g}(M, \Delta)$ :

$$
V_{0}^{r e g}(M, \Delta)=\frac{M^{2}}{4 G_{1}}+\frac{\Delta^{2}}{4 G_{2}}-\int_{0}^{\Lambda} \frac{d p_{1}}{2 \pi}\left(\sqrt{p_{1}^{2}+(M+\Delta)^{2}}+\sqrt{p_{1}^{2}+(M-\Delta)^{2}}\right) .
$$

\footnotetext{
${ }^{6}$ Indeed, if simultaneously with $\mu_{5} \rightarrow-\mu_{5}$ and/or $\mu \rightarrow-\mu$ transformations we perform in the integral 15 the following change of variables, $p_{1} \rightarrow-p_{1}$ and/or $p_{0} \rightarrow-p_{0}$, then one can easily see that the expression (16) remains intact.
} 
Since this expression diverges at $\Lambda \rightarrow \infty$, it is necessary to renormalize it, assuming that $G_{1} \equiv G_{1}(\Lambda)$ and $G_{2} \equiv G_{2}(\Lambda)$ have appropriate $\Lambda$ dependencies. It is easy to establish that if

$$
\frac{1}{4 G_{1}} \equiv \frac{1}{4 G_{1}(\Lambda)}=\frac{1}{2 \pi} \ln \frac{2 \Lambda}{M_{1}}, \quad \frac{1}{4 G_{2}} \equiv \frac{1}{4 G_{2}(\Lambda)}=\frac{1}{2 \pi} \ln \frac{2 \Lambda}{M_{2}},
$$

where $M_{1}$ and $M_{2}$ are some finite and cutoff independent parameters with dimensionality of mass, then integrating in (20) over $p_{1}$ and ignoring there an unessential term $-\Lambda^{2} / 2 \pi$ one can obtain in the limit $\Lambda \rightarrow \infty$ a finite and renormalization invariant expression for the effective potential,

$$
4 \pi V_{0}(M, \Delta)=M^{2} \ln \frac{\left|M^{2}-\Delta^{2}\right|}{M_{1}^{2}}+\Delta^{2} \ln \frac{\left|M^{2}-\Delta^{2}\right|}{M_{2}^{2}}+2 M \Delta \ln \left|\frac{M+\Delta}{M-\Delta}\right|-\Delta^{2}-M^{2} .
$$

Now two remarks are in order. First, since $M_{1}$ and $M_{2}$ can be considered as free model parameters, it is clear that the renormalization procedure of the $\mathrm{NJL}_{2}$ model (1) is accompanied by the dimensional transmutation phenomenon. Indeed, there are two dimensionless bare coupling constants $G_{1,2}$ in the initial unrenormalized expression (19) for $V_{0}^{u n}(M, \Delta)$, whereas after renormalization the effective potential (22) is characterized by two dimensional, $M_{1}$ and $M_{2}$, free model parameters. Moreover, $M_{1}$ and $M_{2}$ are renormalization invariant quantities, i.e. they do not depend on the normalization points. (The physical sense of $M_{1}$ and $M_{2}$ will be discussed below.) Second, the transposition $G_{1} \leftrightarrow G_{2}$ of the bare coupling constants before renormalization is equivalent, as it is clear from (21), to the transposition $M_{1} \leftrightarrow M_{2}$ after renormalization procedure. Hence, the vacuum effective potential $V_{0}(M, \Delta)$ (22) of the model is invariant with respect to the duality transformation (18) which now, i.e. in vacuum, looks like $M_{1} \leftrightarrow M_{2}, M \leftrightarrow \Delta$.

Note also that the effective potential $V_{0}(M, \Delta)$ written in the form (22) has a singularity at $M=\Delta$, which is really fictitious. Indeed, the expression (22) may be presented in an equivalent form that is more convenient for both numerical and analytical investigations:

$$
4 \pi V_{0}(M, \Delta)=\delta \Delta^{2}-\Delta^{2}-M^{2}+(M-\Delta)^{2} \ln \left|\frac{M-\Delta}{M_{1}}\right|+(M+\Delta)^{2} \ln \left(\frac{M+\Delta}{M_{1}}\right),
$$

where

$$
\frac{\delta}{4 \pi} \equiv \frac{1}{4 G_{2}}-\frac{1}{4 G_{1}}=\frac{1}{2 \pi} \ln \frac{M_{1}}{M_{2}} .
$$

The expression (23) is now a smooth function at $M=\Delta$. As it is clear from (23), instead of two massive $M_{1}$ and $M_{2}$ parameters the renormalized model can be characterized by one massive and one dimensionless parameter $M_{1}$ and $\delta$, respectively. (In this case only the partial dimensional transmutation phenomenon takes place.) Just this set of parameters, i.e. $M_{1}$ and $\delta$, was used in early investigations of the initial model (1) at $\mu_{5}=0$ [21]. In spite of the fact that the dual invariance $D$ (18) of the effective potential in the form (23), i.e. its symmetry with respect to simultaneous transformations $M_{1} \leftrightarrow M_{2}$ and $M \leftrightarrow \Delta$, is not so evident as in the form (22), in the following we will treat the model properties in terms of the parameters $M_{1}$ and $\delta$ as well.

So, if $\delta>0$, i.e., as is easily seen from (24) and (21), at $G_{1}>G_{2}$ or $M_{1}>M_{2}$, the global minimum of the effective potential (23) lies at the point $\left(M=M_{1}, \Delta=0\right)$. This means that if interaction in the fermion-antifermion channel is greater than that in the difermion one, then the chiral symmetry of the model is spontaneously broken down and fermions acquire dynamically a nonzero Dirac mass, which is equal just to the free model parameter $M_{1}$. Further, in order to establish the phase structure of the model (or, equivalently, to find the global minimum point of the function $V_{0}(M, \Delta)$ ) at $\delta<0$, i.e. at $G_{1}<G_{2}$, we do not need a straightforward analytical (or numerical) study of the function (23) on the extremum. In this case it is enough to take into account the dual invariance (18) of the TDP (15) (at $\mu=\mu_{5}=0$ it is reduced to a symmetry of the effective potential $V_{0}(M, \Delta)$ with respect to simultaneous permutations $M_{1} \leftrightarrow M_{2}, M \leftrightarrow \Delta$ ) and conclude (see also the discussion just after (18)) that at $\delta<0$ the effective potential (23) has a global minimum at the point $\left(M=0, \Delta=\Delta_{0}\right)$, where $\Delta_{0}=M_{2}=M_{1} \exp (-\delta / 2)$. Since in this case only the difermion condensate, which is equal to $M_{2}$, is nonzero, the fermion number $U(1)$ symmetry is spontaneously broken and the superconducting phase is realized in the model. Hence, the parameter $M_{2}$ is a Majorana mass of fermions, which appears dynamically in superconducting phase of the model.

\section{The case $\mu>0, \mu_{5}>0$ and $T=0$}

Taking into account the expression (B16) (see Appendix B), in this case the unrenormalized TDP (15) can be presented in the following form

$$
\Omega^{u n}(M, \Delta)=\frac{M^{2}}{4 G_{1}}+\frac{\Delta^{2}}{4 G_{2}}-\int_{0}^{\infty} \frac{d p_{1}}{4 \pi}\left\{\left|p_{01}\right|+\left|p_{02}\right|+\left|\bar{p}_{01}\right|+\left|\bar{p}_{02}\right|\right\},
$$

where quasiparticle and quasiantiparticle energies $p_{01}, p_{02}$ and $\bar{p}_{01}, \bar{p}_{02}$, respectively, are presented in (B5). It is shown in Appendix B (see the text below formula $(\overline{B 13})$ ) how one can find the asymptotic expansion of the integrand in 
(25) at $\left|p_{1}\right| \rightarrow \infty$. As a consequence of this prescription we have obtained the asymptotic expansions (B14) and, as a result, the following $\left|p_{1}\right| \rightarrow \infty$ expansion:

$$
\left|p_{01}\right|+\left|p_{02}\right|+\left|\bar{p}_{01}\right|+\left|\bar{p}_{02}\right|=4\left|p_{1}\right|+\frac{2\left(M^{2}+\Delta^{2}\right)}{\left|p_{1}\right|}+\mathcal{O}\left(1 /\left|p_{1}\right|^{2}\right) .
$$

It means that the integral in (25) is an ultraviolet (UV) divergent, so we need to renormalize the TDP $\Omega^{u n}(M, \Delta)$. Using the momentum cutoff regularization scheme, we obtain

$$
\begin{aligned}
\Omega^{r e g}(M, \Delta)=\frac{M^{2}}{4 G_{1}}+\frac{\Delta^{2}}{4 G_{2}} & -\int_{0}^{\Lambda} \frac{d p_{1}}{4 \pi}\left\{\left|p_{01}\right|+\left|p_{02}\right|+\left|\bar{p}_{01}\right|+\left|\bar{p}_{02}\right|\right\} \\
=V_{0}^{r e g}(M, \Delta) & -\int_{0}^{\Lambda} \frac{d p_{1}}{4 \pi}\left(\left|p_{01}\right|+\left|p_{02}\right|+\left|\bar{p}_{01}\right|+\left|\bar{p}_{02}\right|\right. \\
& \left.-2 \sqrt{p_{1}^{2}+(M+\Delta)^{2}}-2 \sqrt{p_{1}^{2}+(M-\Delta)^{2}}\right)
\end{aligned}
$$

where $V_{0}^{r e g}(M, \Delta)$ is given in (20). Note that the leading terms of the asymptotic expansion (26) do not depend on $\mu$ and $\mu_{5}$. So the quantity

$$
\left.\left(\left|p_{01}\right|+\left|p_{02}\right|+\left|\bar{p}_{01}\right|+\left|\bar{p}_{02}\right|\right)\right|_{\mu=0, \mu_{5}=0} \equiv 2 \sqrt{p_{1}^{2}+(M+\Delta)^{2}}+2 \sqrt{p_{1}^{2}+(M-\Delta)^{2}}
$$

has the same asymptotic expansion (26) at $\left|p_{1}\right| \rightarrow \infty$. Hence, the integral term in (28) is a convergent one, and all UV divergences are located in the first term $V_{0}^{r e g}(M, \Delta)$. The UV divergences are eliminated, if the $\Lambda$ dependencies (21) of the bare coupling constants $G_{1}$ and $G_{2}$ are supposed. In this case we have from (28) at $\Lambda \rightarrow \infty$ the following expression for the renormalized TDP:

$$
\Omega^{r e n}(M, \Delta)=V_{0}(M, \Delta)-\int_{0}^{\infty} \frac{d p_{1}}{4 \pi}\left\{\left|p_{01}\right|+\left|p_{02}\right|+\left|\bar{p}_{01}\right|+\left|\bar{p}_{02}\right|-2 \sqrt{p_{1}^{2}+(M+\Delta)^{2}}-2 \sqrt{p_{1}^{2}+(M-\Delta)^{2}}\right\}
$$

where $V_{0}(M, \Delta)$ is the TDP (effective potential) (22)-(23) of the model at $\mu=0$ and $\mu_{5}=0$. Let us denote by $\left(M_{0}, \Delta_{0}\right)$ the global minimum point (GMP) of the TDP (30). Then, investigating the behavior of this point vs $\mu$ and $\mu_{5}$ it is possible to construct the $\left(\mu, \mu_{5}\right)$-phase portrait (diagram) of the model. A numerical algorithm for finding the quasi(anti)particle energies $p_{01}, p_{02}, \bar{p}_{01}$, and $\bar{p}_{02}$ is elaborated in Appendix B. Based on this, it can be shown numerically that GMP of the TDP can never be of the form $\left(M_{0} \neq 0, \Delta_{0} \neq 0\right)$. Hence, at arbitrary fixed values of $M_{1}$ and $M_{2}$, i.e. at arbitrary values of $\delta(\underline{24})$, it is enough to study the projections $F_{1}(M) \equiv \Omega^{\text {ren }}(M, \Delta=0)$ and $F_{2}(\Delta) \equiv \Omega^{r e n}(M=0, \Delta)$ of the TDP (30) to the $M$ and $\Delta$ axes, correspondingly. Taking into account the relations (B18) and (B19) for the sum $\left|p_{01}\right|+\left|\underline{p_{02} \mid}+\right| \bar{p}_{01}|+| \bar{p}_{02} \mid$ at $\Delta=0$ or $M=0$, it is possible to obtain the following expressions for these quantities,

$$
\begin{aligned}
& F_{1}(M)=-\frac{\mu_{5}^{2}}{2 \pi}-\frac{M^{2}}{4 \pi}+\frac{M^{2}}{2 \pi} \ln \left(\frac{M}{M_{1}}\right)-\frac{\theta(\mu-M)}{2 \pi}\left(\mu \sqrt{\mu^{2}-M^{2}}-M^{2} \ln \frac{\mu+\sqrt{\mu^{2}-M^{2}}}{M}\right) \\
& F_{2}(\Delta)=-\frac{\mu^{2}}{2 \pi}-\frac{\Delta^{2}}{4 \pi}+\frac{\Delta^{2}}{2 \pi} \ln \left(\frac{\Delta}{M_{2}}\right)-\frac{\theta\left(\mu_{5}-\Delta\right)}{2 \pi}\left(\mu_{5} \sqrt{\mu_{5}^{2}-\Delta^{2}}-\Delta^{2} \ln \frac{\mu_{5}+\sqrt{\mu_{5}^{2}-\Delta^{2}}}{\Delta}\right) .
\end{aligned}
$$

(Details of the derivation of these expressions are given in Appendix C.) Now, to find the GMP of the whole TDP (30) and, as a consequence, to obtain the phase structure of the model, it is sufficient to compare the minimal values of the functions (31) and (32). Recall that, up to an unessential constant, each of the functions $F_{1}(M)$ and $F_{2}(\Delta)$ is just a well-known TDP of the usual massless Gross-Neveu model at zero temperature and nonzero chemical potential. It was investigated, e.g., in [34]. So, one can conclude that at $\mu<\mu_{c} \equiv M_{1} / \sqrt{2}\left(\mu_{5}<\mu_{5 c} \equiv M_{2} / \sqrt{2}\right)$ the GMP of the function $F_{1}(M)$ (of the function $F_{2}(\Delta)$ ) lies at the point $M=M_{1}$ (at the point $\Delta=M_{2}$ ). Whereas at $\mu>\mu_{c}($ at $\left.\mu_{5}>\mu_{5 c}\right)$ the GMP is at the point $M=0(\Delta=0)$. Moreover, the corresponding minimal values are the following:

$$
F_{1}\left(M_{1}\right)=-\frac{\mu_{5}^{2}}{2 \pi}-\frac{M_{1}^{2}}{4 \pi}, \quad F_{2}\left(M_{2}\right)=-\frac{\mu^{2}}{2 \pi}-\frac{M_{2}^{2}}{4 \pi}, \quad F_{1}(0)=F_{2}(0)=-\frac{\mu_{5}^{2}}{2 \pi}-\frac{\mu^{2}}{2 \pi} .
$$

Comparing the least values (33) of the TDPs (31) and (32) for different values of the chemical potentials $\mu$ and $\mu_{5}$, it is possible to obtain the $\left(\mu, \mu_{5}\right)$-phase portrait of the model, which consists of only three phases, the chiral symmetry breaking phase, the superconducting phase and, finally, symmetrical phase. Moreover, it is evident that in the CSB phase the GMP of the TDP (30) has the form $\left(M_{1}, 0\right)$, and in the SC phase it lies at the point $\left(0, M_{2}\right)$, whereas in the symmetrical phase the least value of the TDP (30) is reached at the point $(M=0, \Delta=0)$. Note that the phase structure of the model depends essentially on the relation between $M_{1}$ and $M_{2}$. Indeed, let us first suppose that $M_{1}>M_{2}$. In this case the typical $\left(\mu, \mu_{5}\right)$-phase portrait of the model is presented in Fig. 1 . It is evident that the region $\left\{\mu>\mu_{c}, \mu_{5}>\mu_{5 c}\right\}$ of the figure corresponds to the symmetrical phase of the model. Moreover, in the region 


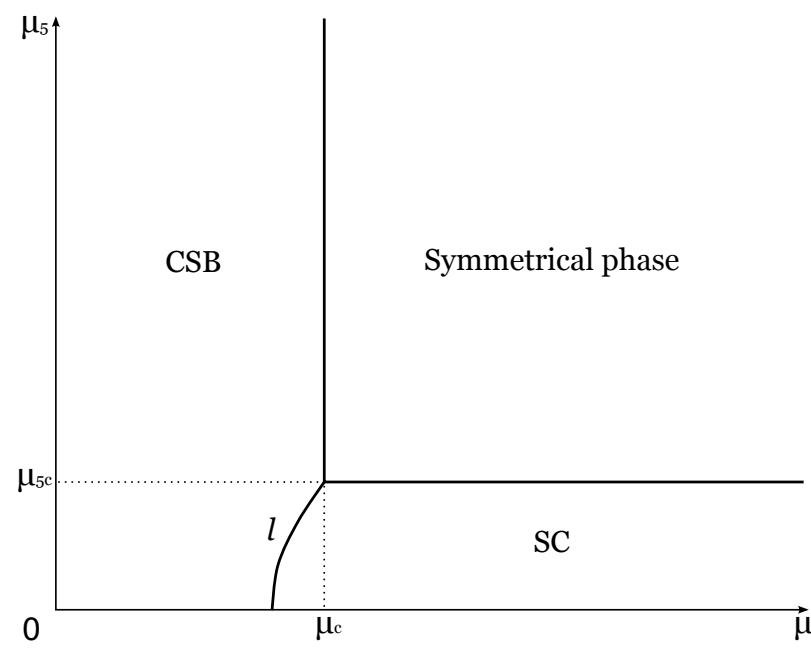

FIG. 1. The typical $\left(\mu, \mu_{5}\right)$-phase structure of the model in the homogeneous case of the ansatz (10) for condensates $\left(b=0, b^{\prime}=0\right)$, when $M_{1}>M_{2}$. The notations CSB and SC are used for the chiral symmetry breaking and superconducting phases, respectively. $\mu_{c}=M_{1} / \sqrt{2}$, $\mu_{5 c}=M_{2} / \sqrt{2}$. The boundary $l$ between CSB and SC phases is defined by (35).

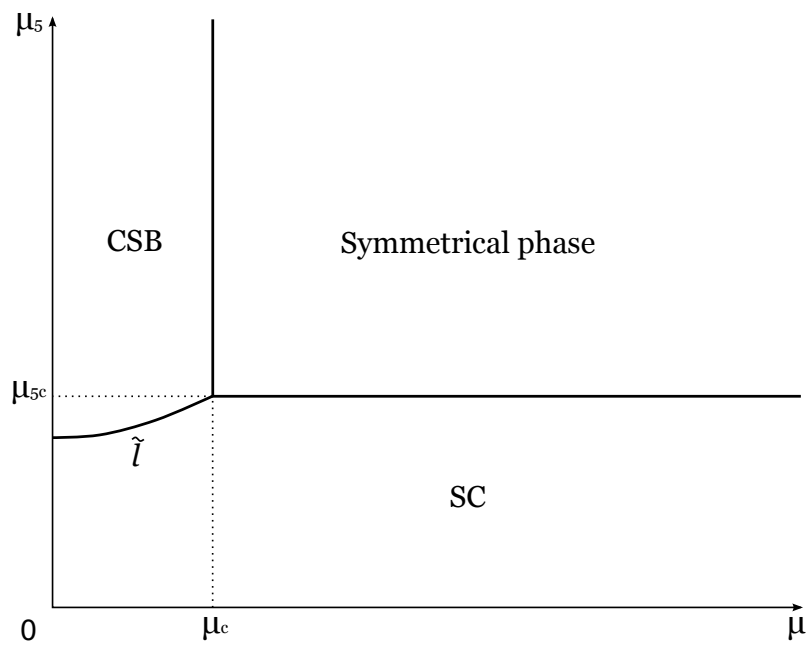

FIG. 2. The typical $\left(\mu, \mu_{5}\right)$-phase structure of the model in the homogeneous case of the ansatz (10) for condensates $\left(b=0, b^{\prime}=0\right)$, when $M_{1}<M_{2}$. The notations CSB and SC are used for the chiral symmetry breaking and superconducting phases, respectively. $\mu_{c}=M_{1} / \sqrt{2}$, $\mu_{5 c}=M_{2} / \sqrt{2}$. The boundary $\tilde{l}$ between CSB and SC phases is defined by (36).

$\left\{\mu<\mu_{c}, \mu_{5}>\mu_{5 c}\right\}$ (in the region $\left\{\mu>\mu_{c}, \mu_{5}<\mu_{5 c}\right\}$ ) of the figure the CSB phase (the SC phase) is arranged. The competition between CSB and SC phases takes place in the region $\left\{\mu<\mu_{c}, \mu_{5}<\mu_{5 c}\right\}$. Namely, the critical curve $l$ of Fig. 1 is defined by the equation $F_{1}\left(M_{1}\right)=F_{2}\left(M_{2}\right)$, i.e. by the equation

$$
\Omega^{r e n}\left(M=M_{1}, \Delta=0\right)=\Omega^{r e n}\left(M=0, \Delta=M_{2}\right) .
$$

The curve $l$ divides this region into two subregions. To the left of $l$ the CSB phase is arranged, whereas to the right of $l$ we have the SC phase. Furthermore, it is clear from (34) and (33) that it is possible to obtain an exact analytical expression for $l$,

$$
l=\left\{\left(\mu, \mu_{5}\right): \mu=\sqrt{\mu_{5}^{2}+\frac{M_{1}^{2}-M_{2}^{2}}{2}}\right\} .
$$

In a similar way it is possible to construct a $\left(\mu, \mu_{5}\right)$-phase portrait of the model when $M_{1}<M_{2}$ (the typical $\left(\mu, \mu_{5}\right)$-phase portrait is presented in Fig. 2). The critical curve $\tilde{l}$ of the figure is given by the relation

$$
\tilde{l}=\left\{\left(\mu, \mu_{5}\right): \mu_{5}=\sqrt{\mu^{2}+\frac{M_{2}^{2}-M_{1}^{2}}{2}}\right\} .
$$

Finally, if $M_{1}=M_{2}$, then the typical $\left(\mu, \mu_{5}\right)$-phase portrait of the model is given in Fig. 3 .

Suppose that the values of $M_{1}$ and $M_{2}$, for which the phase portrait of Fig. 2 is drawn, are obtained by rearrangement of the corresponding $M_{1}, M_{2}$ values for which Fig. 1 is depicted (and vice versa). For example, let us assume that $M_{1}=m_{1}, M_{2}=m_{2}\left(m_{1}>m_{2}\right)$ in Fig. 1, but Fig. 2 is obtained for values $M_{1}=m_{2}$ and $M_{2}=m_{1}$. Then it is easy to show that Figs. 1 and 2 are dually connected; i.e. Fig. 2 can be obtained from Fig. 1 by applying the duality transformation $D$ (18) (and vice versa). Indeed, the transformation $D$ can be divided into three more simple steps. i) First, performing the $\mu \leftrightarrow \mu_{5}$ transformation in Fig. 1, we rename the coordinate axes of the figure. ii) Second, when the coordinates of the GMP are transposed, i.e. $M_{0} \leftrightarrow \Delta_{0}$, we have renaming of the phases. (For example, in this case the GMP of the CSB phase, i.e. the point $\left(M_{1}, 0\right)$, is transformed into the point $\left(0, M_{1}\right)$ and, as a result, the CSB phase is transformed into the SC phase.) iii) Finally, performing the transposition $M_{1} \leftrightarrow M_{2}$ (which corresponds to $G_{1} \leftrightarrow G_{2}$ of (18)) and directing vertically (horizontally) the $\mu_{5}$ axis (the $\mu$ axis), we obtain just Fig. 2, corresponding to $M_{2}=m_{1}>M_{1}=m_{2}$.

It is interesting to note that at $M_{1}=M_{2}$, i.e. at $G_{1}=G_{2}$ or $\delta=0$, the phase portrait of the model (see Fig. 3) is dually invariant, or self-dual. Moreover, in spite of self-duality at $G_{1}=G_{2}$ of the phase structure of the model, the CSB and SC ground states are not degenerate in this case. Indeed, at $\mu_{5}>\mu$ the CSB phase is preferable, but at $\mu_{5}<\mu$ the ground state of the SC phase has a lower energy (at $\mu<\mu_{c}$ ). The degeneracy between ground states of these phases occurs in this case only at the critical curve $L$ (see Fig. 3), where $\mu=\mu_{5}$. 


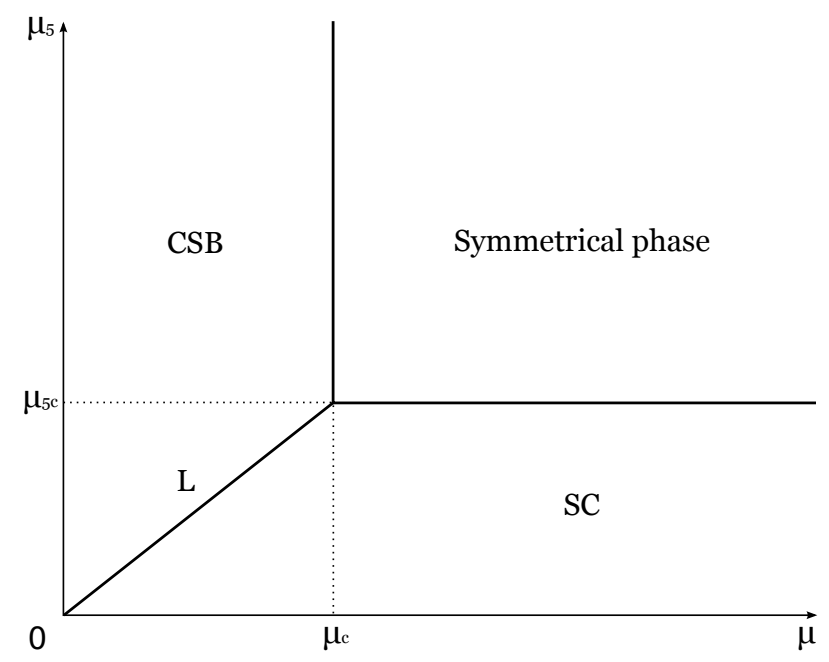

FIG. 3. The typical $\left(\mu, \mu_{5}\right)$-phase structure of the model in the homogeneous case of the ansatz (10) for condensates $(b=0$, $\left.b^{\prime}=0\right)$, when $M_{1}=M_{2}$. All the notations are the same as in Figs 1, 2 except the boundary L between CSB and SC phases, which is defined by the relation $\mu=\mu_{5}$.

The knowledge of the GMP $\left(M_{0}, \Delta_{0}\right)$ of the TDP (30) provides us with particle number density $n$ and chiral charge density $n_{5}$ :

$$
n=-\frac{\partial \Omega^{r e n}\left(M_{0}, \Delta_{0}\right)}{\partial \mu}, \quad n_{5}=-\frac{\partial \Omega^{r e n}\left(M_{0}, \Delta_{0}\right)}{\partial \mu_{5}} .
$$

So, to obtain the behavior of these quantities in the symmetrical, CSB and SC phases of Figs 1, 2 one can use directly the least values (33) of the TDP (32) in these phases. For example, we have for densities $n$ and $n_{5}$ in the CSB phase:

$$
\left.n\right|_{\mathrm{CSB}}=-\frac{\partial F_{1}\left(M_{1}\right)}{\partial \mu} \equiv 0,\left.n_{5}\right|_{\mathrm{CSB}}=-\frac{\partial F_{1}\left(M_{1}\right)}{\partial \mu_{5}}=\frac{\mu_{5}}{\pi} .
$$

By analogy, in the SC and symmetrical (SYM) phases we have for densities $n$ and $n_{5}$

$$
\left.n_{5}\right|_{\mathrm{SC}} \equiv 0,\left.n\right|_{\mathrm{SC}}=\frac{\mu}{\pi} ;\left.n\right|_{\mathrm{SYM}}=\frac{\mu}{\pi},\left.n_{5}\right|_{\mathrm{SYM}}=\frac{\mu_{5}}{\pi} .
$$

It is clear from (38) that at $M_{1}>M_{2}$ (or at $G_{1}>G_{2}$ ) the CSB phase is realized at sufficiently small values of $\mu$ for arbitrary values of $\mu_{5}$ (see Fig. 1). Hence, in this case and under a supposition of a spatially homogeneous structure of the condensates the particle density $n$ of the system is always equal to zero at sufficiently low values of chemical potential $\mu$. Correspondingly, in the case $M_{1}<M_{2}$ (or at $G_{1}<G_{2}$ ) the chiral charge density $n_{5}$ is equal to zero at sufficiently small values of $\mu_{5}$ and for arbitrary values of $\mu$ (see Fig. 2 and (39)).

On the basis of the obtained results, we study in the next section the phase structure of the model when condensates are allowed to be inhomogeneous in the framework of the ansatz (10).

\section{INHOMOGENEOUS CASE OF THE ANSATZ (10): $b \neq 0$ AND $b^{\prime} \neq 0$}

\section{A. Renormalization procedure}

Taking into account the results of the Appendix B, it is evident that in the case under consideration the unrenormalized TDP (14) can be obtained from the TDP (25), corresponding to the case $b=0$ and $b^{\prime}=0$, by simple replacements, $\mu \rightarrow \tilde{\mu} \equiv \mu-b$ and $\mu_{5} \rightarrow \tilde{\mu}_{5} \equiv \mu_{5}-b^{\prime}$. So we have

$$
\Omega^{u n}\left(M, b, b^{\prime}, \Delta\right)=\frac{M^{2}}{4 G_{1}}+\frac{\Delta^{2}}{4 G_{2}}-\Omega_{1}-\Omega_{2}-\Omega_{3}-\Omega_{4},
$$

where

$$
\Omega_{1}=\int_{0}^{\infty} \frac{d p_{1}}{4 \pi}\left|P_{01}\right|, \Omega_{2}=\int_{0}^{\infty} \frac{d p_{1}}{4 \pi}\left|P_{02}\right|, \Omega_{3}=\int_{0}^{\infty} \frac{d p_{1}}{4 \pi}\left|\bar{P}_{01}\right|, \Omega_{4}=\int_{0}^{\infty} \frac{d p_{1}}{4 \pi}\left|\bar{P}_{02}\right|,
$$

and the quantities $P_{01}, P_{02}, \bar{P}_{01}, \bar{P}_{02}$ are now the quasiparticle energies (B5), in which the above-mentioned changes of the chemical potentials should be done, $\mu \rightarrow \tilde{\mu}$ and $\mu_{5} \rightarrow \tilde{\mu}_{5}$. As it follows from the discussion below (17), it is 
sufficient to study the TDP (40) at $M \geq 0, \Delta \geq 0, \tilde{\mu} \geq 0$, and $\tilde{\mu}_{5} \geq 0$. Moreover, the TDP (40) is invariant with respect to the duality transformation $\tilde{D}$ :

$$
\tilde{D}: \quad G_{1} \longleftrightarrow G_{2}, \quad \mu \longleftrightarrow \mu_{5}, \quad M \longleftrightarrow \Delta, \quad b \longleftrightarrow b^{\prime}
$$

(Recall that after the renormalization procedure the transposition $G_{1} \leftrightarrow G_{2}$ is equivalent to $M_{1} \leftrightarrow M_{2}$, or to changing the sign of the parameter $\delta(24), \delta \rightarrow-\delta$.)

To find a finite renormalized expression for the TDP (40), we should first regularize it and then perform a renormalization procedure in order to remove at $\Lambda \rightarrow \infty$ the UV divergences by demanding an appropriate behavior of the bare coupling constants $G_{1,2}$ vs the cutoff parameter $\Lambda$. In the case of spatially homogeneous condensates all regularization schemes are usually equivalent. However, in the case of spatially inhomogeneous condensates the translational invariance over one or several spatial coordinates is lost. So, the corresponding (spatial) momenta are not conserved. Then, if one uses the momentum-cutoff regularization technique, as in the previous section, nonphysical (spurious) $b, b^{\prime}$-dependent terms appear, and the TDP acquires some nonphysical properties such as unboundedness from below with respect to $b, b^{\prime}$, etc. In order to obtain a physically reliable TDP (or effective potential), in this case an additional substraction procedure is usually applied (for details see [9, 19]). On the other hand, if one uses more adequate regularization schemes such as Schwinger proper-time [2, 4, 5] or energy-cutoff regularizations [10, 20], etc., such spurious terms do not appear. 7

In the present paper the energy cutoff regularization scheme of [10] is adopted. (See also [19, 20, 35, 36], where a similar regularization was used in searching for chiral density waves and inhomogeneous charged pion and Cooper condensates in some $\mathrm{NJL}_{2}$ models.) Namely, we require that only quasiparticle energies with momenta $p_{1}$, constrained by the relations

$$
\left|P_{01}\right|<\Lambda,\left|P_{02}\right|<\Lambda,\left|\bar{P}_{01}\right|<\Lambda,\left|\bar{P}_{02}\right|<\Lambda,
$$

contribute to the regularized expressions of the integrals (41) in $\Omega_{1}, \ldots, \Omega_{4}$, correspondingly. At sufficiently high values of the cutoff $\Lambda$ it is possible to use in (43) only the leading terms of the asymptotic relations (B14) for $\left|P_{01}\right|, P_{02} \mid$, $\left|\bar{P}_{01}\right|$, and $\left|\bar{P}_{02}\right|$. As a result, we have the following expressions, regularized in the framework of the energy cutoff scheme:

$$
\begin{aligned}
& \Omega_{1}^{r e g}=\int_{0}^{\Lambda+\tilde{\mu}-\tilde{\mu}_{5}} \frac{d p_{1}}{4 \pi}\left|P_{01}\right|=\int_{0}^{\Lambda} \frac{d p_{1}}{4 \pi}\left|P_{01}\right|+\int_{\Lambda}^{\Lambda+\tilde{\mu}-\tilde{\mu}_{5}} \frac{d p_{1}}{4 \pi}\left|P_{01}\right|, \\
& \Omega_{2}^{r e g}=\int_{0}^{\Lambda-\tilde{\mu}+\tilde{\mu}_{5}} \frac{d p_{1}}{4 \pi}\left|P_{02}\right|=\int_{0}^{\Lambda} \frac{d p_{1}}{4 \pi}\left|P_{02}\right|+\int_{\Lambda}^{\Lambda-\tilde{\mu}+\tilde{\mu}_{5}} \frac{d p_{1}}{4 \pi}\left|P_{02}\right|, \\
& \Omega_{3}^{r e g}=\int_{0}^{\Lambda-\tilde{\mu}-\tilde{\mu}_{5}} \frac{d p_{1}}{4 \pi}\left|\bar{P}_{01}\right|=\int_{0}^{\Lambda} \frac{d p_{1}}{4 \pi}\left|\bar{P}_{01}\right|+\int_{\Lambda}^{\Lambda-\tilde{\mu}-\tilde{\mu}_{5}} \frac{d p_{1}}{4 \pi}\left|\bar{P}_{01}\right|, \\
& \Omega_{4}^{r e g}=\int_{0}^{\Lambda+\tilde{\mu}+\tilde{\mu}_{5}} \frac{d p_{1}}{4 \pi}\left|\bar{P}_{02}\right|=\int_{0}^{\Lambda} \frac{d p_{1}}{4 \pi}\left|\bar{P}_{02}\right|+\int_{\Lambda}^{\Lambda+\tilde{\mu}+\tilde{\mu}_{5}} \frac{d p_{1}}{4 \pi}\left|\bar{P}_{02}\right| .
\end{aligned}
$$

Using these expressions instead of $\Omega_{i}$ in (40) $(i=1, \ldots, 4)$, one can obtain the following regularized TDP,

$$
\begin{aligned}
\Omega^{r e g}\left(M, b, b^{\prime}, \Delta\right)=\widetilde{\Omega}^{r e g}(M, \Delta) & -\int_{\Lambda}^{\Lambda+\tilde{\mu}-\tilde{\mu}_{5}} \frac{d p_{1}}{4 \pi}\left|P_{01}\right|-\int_{\Lambda}^{\Lambda-\tilde{\mu}+\tilde{\mu}_{5}} \frac{d p_{1}}{4 \pi}\left|P_{02}\right| \\
& -\int_{\Lambda}^{\Lambda-\tilde{\mu}-\tilde{\mu}_{5}} \frac{d p_{1}}{4 \pi}\left|\bar{P}_{01}\right|-\int_{\Lambda}^{\Lambda+\tilde{\mu}+\tilde{\mu}_{5}} \frac{d p_{1}}{4 \pi}\left|\bar{P}_{02}\right|,
\end{aligned}
$$

where $\widetilde{\Omega}^{r e g}(M, \Delta)$ is the TDP (27) of the previous section, regularized by a momentum cutoff approach, in which the replacements $\mu \rightarrow \tilde{\mu}$ and $\mu_{5} \rightarrow \tilde{\mu}_{5}$ should be performed. It is evident that in the limit $\Lambda \rightarrow \infty$ we obtain from $\widetilde{\Omega}^{r e g}(M, \Delta)$ the renormalized TDP $\widetilde{\Omega}^{r e n}(M, \Delta)$ which is the TDP (30), obtained for the case of homogeneous condensates with $\mu \rightarrow \tilde{\mu}$ and $\mu_{5} \rightarrow \tilde{\mu}_{5}$. So, in the limit $\Lambda \rightarrow \infty$ we get from (45) the following expression for the renormalized TDP in the case of inhomogeneous condensates,

$$
\Omega^{r e n}\left(M, b, b^{\prime}, \Delta\right)=\widetilde{\Omega}^{r e n}(M, \Delta)+\frac{\tilde{\mu}^{2}}{2 \pi}+\frac{\tilde{\mu}_{5}^{2}}{2 \pi}-\frac{\mu^{2}}{2 \pi}-\frac{\mu_{5}^{2}}{2 \pi} .
$$

(To obtain the second and third terms in the right-hand side of (46), one should take into account that at $\Lambda \rightarrow \infty$ it is possible to use in (45) the asymptotic expansions (B14) for the integrand functions $P_{01}, P_{02}, \bar{P}_{01}, \bar{P}_{02}$. Then the integration can be easily done. Moreover, we also add to the expression (46) unessential $b, b^{\prime}$-independent terms, $-\mu^{2} / 2 \pi$ and $-\mu_{5}^{2} / 2 \pi$, in order to reproduce at $b, b^{\prime}=0$ the TDP (30), corresponding to a spatially homogeneous chiral condensate. 8)

\footnotetext{
7 As discussed in the recent papers [2, [5, 10, 19, 20], an adequate regularization scheme in the case of spatially inhomogeneous phases consists in the following: for different quasiparticles the same restriction on their region of energy values $\left|p_{01}\right|, \ldots,\left|\bar{p}_{02}\right|$ should be used in a regularized thermodynamic potential.

${ }^{8}$ In fact these summands may be obtained as a result of subtracting the terms with $b=0$ and $b^{\prime}=0$ in evaluating Eqs. B16) with the
} 


\section{B. Phase structure at $T=0$}

It is clear that to find the phase portrait of the model at $T=0$, one should investigate the global minimum point (GMP) of the TDP $\Omega^{r e n}\left(M, b, b^{\prime}, \Delta\right)$ (46) vs the dynamical variables $M, b, b^{\prime}, \Delta$. Since in our case the variables $b$ and $b^{\prime}$ are absorbed by the chemical potentials $\mu$ and $\mu_{5}$, the TDP (46) is indeed a function of four variables $M, \Delta$, $\tilde{\mu} \equiv \mu-b$ and $\tilde{\mu}_{5} \equiv \mu_{5}-b^{\prime}$. Thus, searching for the GMP of this function consists effectively of two stages. First, one can find the extremum of this function over $M \geq 0$ and $\Delta \geq 0$ (taking into account the results of Sec. IIIC) 9 and then one should minimize the obtained expression over the variables $\tilde{\mu} \geq 0, \tilde{\mu}_{5} \geq 0$. Following this strategy, let us introduce for arbitrary fixed values of the usual chemical potentials $\mu$ and $\mu_{5}$ the quantity

$$
\omega\left(\tilde{\mu}, \tilde{\mu}_{5}\right)=\min _{M \geq 0, \Delta \geq 0}\left\{\Omega^{r e n}\left(M, b, b^{\prime}, \Delta\right)\right\} .
$$

Taking into account the results of the investigation of the GMP of the TDP (30) (see Sec. IIIC), it is easy to show that if a point $\left(\tilde{\mu}, \tilde{\mu}_{5}\right)$ belongs to the CSB regions of the $\left(\tilde{\mu}, \tilde{\mu}_{5}\right)$ plane (see, e.g, Fig. 1 with replacements $\mu \rightarrow \tilde{\mu}$ and $\left.\mu_{5} \rightarrow \tilde{\mu}_{5}\right)$, then, as it follows from (33), we have for the function (47)

$$
\left.\omega\left(\tilde{\mu}, \tilde{\mu}_{5}\right)\right|_{\mathrm{CSB}}=\frac{\tilde{\mu}^{2}}{2 \pi}-\frac{M_{1}^{2}}{4 \pi}-\frac{\mu^{2}}{2 \pi}-\frac{\mu_{5}^{2}}{2 \pi} .
$$

In a similar way, it is easily seen from (47), (46) and (33) that if a point $\left(\tilde{\mu}, \tilde{\mu}_{5}\right)$ lies in the SC or symmetrical region of the above-mentioned $\left(\tilde{\mu}, \tilde{\mu}_{5}\right)$ plane, then the function $\omega\left(\tilde{\mu}, \tilde{\mu}_{5}\right)$ is reduced to the expressions

$$
\left.\omega\left(\tilde{\mu}, \tilde{\mu}_{5}\right)\right|_{\mathrm{SC}}=\frac{\tilde{\mu}_{5}^{2}}{2 \pi}-\frac{M_{2}^{2}}{4 \pi}-\frac{\mu^{2}}{2 \pi}-\frac{\mu_{5}^{2}}{2 \pi},\left.\omega\left(\tilde{\mu}, \tilde{\mu}_{5}\right)\right|_{\mathrm{SYM}}=-\frac{\mu^{2}}{2 \pi}-\frac{\mu_{5}^{2}}{2 \pi},
$$

correspondingly. (Note that in (48) and (49) the chemical potentials without tildes, $\mu$ and $\mu_{5}$, are some fixed external parameters.) It is evident that the function $\omega\left(\tilde{\mu}, \tilde{\mu}_{5}\right)$, presented by the expressions (48) and (49), is a continuous one in the region $\tilde{\mu} \geq 0, \tilde{\mu}_{5} \geq 0$. Further, to find the least value of this function over variables $\tilde{\mu} \geq 0$ and $\tilde{\mu}_{5} \geq 0$ as well as the points where it is achieved, we consider three qualitatively different cases, (i) $M_{1}>M_{2}$, (ii) $M_{1}<M_{2}$, and (iii) $M_{1}=M_{2}$.

(i) The case $M_{1}>M_{2}\left(G_{1}>G_{2}\right)$. In this case it is easy to see from the relations (48)-(49) that the function (47) $\omega\left(\tilde{\mu}, \tilde{\mu}_{5}\right)$ reaches its minimal value on the $\tilde{\mu}_{5}$ axis, i.e. at $\tilde{\mu} \equiv \mu-b=0$. The set of these points lies in the CSB region of the $\left(\tilde{\mu}, \tilde{\mu}_{5}\right)$ plane corresponding to the $\left(M_{0}=M_{1}, \Delta_{0}=0\right)$-extreme point of the TDP (30). Since in this case the modulus of the difermion condensate is equal to zero, $\Delta_{0}=0$, we are free to put $b^{\prime}=0$, i.e. $\tilde{\mu}_{5}=\mu_{5}$. Hence, at $M_{1}>M_{2}$ and at arbitrary fixed values of chemical potentials $\mu \geq 0, \mu_{5} \geq 0$ the global minimum of the TDP (46) $\Omega^{r e n}\left(M, b, b^{\prime}, \Delta\right)$ is arranged at the point $\left(M=M_{1}, b=\mu, b^{\prime}=0, \Delta=0\right)$, where

$$
\Omega^{r e n}\left(M=M_{1}, b=\mu, b^{\prime}=0, \Delta=0\right)=\omega\left(\tilde{\mu}=0, \tilde{\mu}_{5}=\mu_{5}\right)=-\frac{M_{1}^{2}}{4 \pi}-\frac{\mu^{2}}{2 \pi}-\frac{\mu_{5}^{2}}{2 \pi} .
$$

As a result, one can see that for arbitrary values of $\mu \geq 0, \mu_{5} \geq 0$ the spatially inhomogeneous phase in the form of chiral spirals (chiral density waves) is more preferable in the model than any of the three homogeneous phases (symmetrical, homogeneous chiral symmetry breaking and homogeneous superconducting phases) or inhomogeneous superconducting phase.

Taking into account the definitions of the particle number density $n$ and chiral charge density $n_{5}$ (37), it is possible, using the least value (50) of the TDP (46), to find these quantities in the inhomogeneous chiral density wave phase. Namely, we have in this phase

$$
n=\frac{\mu}{\pi}, \quad n_{5}=\frac{\mu_{5}}{\pi} .
$$

Let us compare the relations (51) with expressions (38)-(39), obtained for $n$ and $n_{5}$ densities in the case of homogeneous condensates. We see that at $M_{1}>M_{2}$ and in the supposition of spatially homogeneous condensates the particle density $n$ of the system always vanishes in the CSB phase, i.e. at sufficiently small values of $\mu$ (see Fig. 1 and (38)). In contrast, if spatial inhomogeneity of condensates is allowed in the framework of the model $(1)$ at $\delta>0$, then in its ground state, corresponding to a chiral density wave phase (at arbitrary values of $\mu>0$ and $\mu_{5}>0$ ), a nonzero particle density $n$ is generated in the system even at infinitesimal values of $\mu$, as it follows from (51).

(ii) The case $M_{1}<M_{2}\left(G_{1}<G_{2}\right)$. There is no need to study the phase structure of the model in this case as detailed as at $M_{1}>M_{2}$, because the phase structure of the model at $G_{1}<G_{2}$ can be obtained using the invariance of

help of a symbolic formula (B15), which is itself defined up to an appropriate subtraction (see, e.g. [37], p. 248). On the other hand, it is obvious that such a "by-hand" addition of last two terms in 46 does not influence the phase structure of the model. However, we guess that this "by-hand" subtracting procedure could be avoided in the framework of our approach only in the case of taking into account the factor arising in the path integral (11)-12) after the Weinberg transformation (see the comments in the footnote 4). This factor could have an appropriate $\mu$ and $\mu_{5}$ dependence in order to reproduce the correct expression (46) for the TDP.

9 As in the case with $b=0$ and $b^{\prime}=0$, in the inhomogeneous case we could not find local minimum points of the TDP 46], in which both $M \neq 0$ and $\Delta \neq 0$. 
the TDP (46) with respect to the duality transformation (42). Indeed, at $M_{1}>M_{2}$, i.e. at $G_{1}>G_{2}$, the TDP (46) has a global minimum at the point of the form $\left(M=M_{1}, b=\mu, b^{\prime}=0, \Delta=0\right)$. Applying the duality transformation (42) to this TDP, i.e. performing the replacements $G_{1} \leftrightarrow G_{2}$ or $M_{1} \leftrightarrow M_{2}, b \leftrightarrow b^{\prime}$, etc., we obtain the TDP, corresponding to the case $M_{1}<M_{2}$, whose least value is achieved at the point $\left(M=0, b=0, b^{\prime}=\mu_{5}, \Delta=M_{2}\right)$, corresponding to a ground state of the inhomogeneous superconducting phase. Hence, at $M_{1}<M_{2}$ for arbitrary values of $\mu>0$ and $\mu_{5}>0$ the nonuniform SC phase is realized in the model. The expressions for particle density $n$ and chiral charge density $n_{5}$ in this phase are still represented by the relations (51).

Recall that in the case of a homogeneous ansatz for condensates and at $M_{1}<M_{2}$ the superconducting phase with $n_{5}=0$ is arranged at rather small values of an axial chemical potential $\mu_{5}$ (see Fig. 2). However, if the possibility of spatial inhomogeneous condensates in the form (10) is taken into account, then at $M_{1}<M_{2}$ the nonuniform superconducting phase is realized, in which $n_{5} \neq 0$ even at arbitrary low values of $\mu_{5}$.

(iii) The case $M_{1}=M_{2}\left(G_{1}=G_{2}\right)$. In this case, using the technique of point $\left.\mathbf{i}\right)$, it is possible to show that at arbitrary fixed $\mu>0$ and $\mu_{5}>0$ the TDP (46), $\Omega^{r e n}\left(M, b, b^{\prime}, \Delta\right)$, has a degenerated least value, which is reached in two different points, $\left(M=M_{1}, b=\mu, b^{\prime}=0, \Delta=0\right)$ and $\left(M=0, b=0, b^{\prime}=\mu_{5}, \Delta=M_{1}\right)$, corresponding to ground state expectation values of inhomogeneous chiral symmetry breaking and superconducting phases. It means that at $M_{1}=M_{2}$ there is a degeneracy between inhomogeneous chiral symmetry breaking and inhomogeneous superconductivity in the whole $\left(\mu, \mu_{5}\right)$ plane. In contrast, in the homogeneous case of the ansatz (10) for condensates a degeneracy between spatially uniform CSB and SC phases is absent, except the line $\mu=\mu_{5}$ of this plane, where $\mu<M_{1} / \sqrt{2}$.

The degeneracy of these ground states means that for arbitrary fixed values of chemical potentials $\mu>0$ and $\mu_{5}>0$ in the space, filled with the chiral density wave phase, a bubble of the inhomogeneous superconducting phase (and vice versa) can be created.

\section{Phase structure at $T>0$}

To introduce finite temperature into the above consideration, it is very convenient to use the following representation of the unrenormalized TDP (40):

$$
\Omega^{u n}\left(M, b, b^{\prime}, \Delta\right)=\frac{M^{2}}{4 G_{1}}+\frac{\Delta^{2}}{4 G_{2}}+\frac{i}{2} \int \frac{d^{2} p}{(2 \pi)^{2}} \ln \left[\left(p_{0}-P_{01}\right)\left(p_{0}-P_{02}\right)\left(p_{0}-\bar{P}_{01}\right)\left(p_{0}-\bar{P}_{02}\right)\right] .
$$

(Integrating in (52) over $p_{0}$ with the help of relation (B15), one obtains the expression (40) for the unrenormalized TDP.) Then, to find the temperature-dependent unrenormalized TDP $\Omega_{T}^{u n}\left(M, b, b^{\prime}, \Delta\right)$ one should replace in (52) the integration over $p_{0}$ in favor of the summation over Matsubara frequencies $\omega_{n}$ by the rule

$$
\int_{-\infty}^{\infty} \frac{d p_{0}}{2 \pi}(\cdots) \rightarrow i T \sum_{n=-\infty}^{\infty}(\cdots), \quad p_{0} \rightarrow p_{0 n} \equiv i \omega_{n} \equiv i \pi T(2 n+1), \quad n=0, \pm 1, \pm 2, \ldots
$$

Summing over Matsubara frequencies in the obtained expression (see e.g. [38] and Appendix [D), we have

$$
\begin{aligned}
\Omega_{T}^{u n}\left(M, b, b^{\prime}, \Delta\right)= & \frac{M^{2}}{4 G_{1}}+\frac{\Delta^{2}}{4 G_{2}}-\int_{0}^{\infty} \frac{d p_{1}}{4 \pi}\left\{\left|P_{01}\right|+\left|P_{02}\right|+\left|\bar{P}_{01}\right|+\left|\bar{P}_{02}\right|\right\} \\
& -T \int_{0}^{\infty} \frac{d p_{1}}{2 \pi} \ln \left\{\left[1+e^{-\beta\left|P_{01}\right|}\right]\left[1+e^{-\beta\left|P_{02}\right|}\right]\left[1+e^{-\beta\left|\bar{P}_{01}\right|}\right]\left[1+e^{-\beta\left|\bar{P}_{02}\right|}\right]\right\},
\end{aligned}
$$

where $\beta=1 / T$. The last integral in (54) is a convergent one, whereas other terms form the zero temperature unrenormalized TDP (40). Hence, it is sufficient to renormalize just this component of the whole TDP (54), using the energy-cutoff regularization scheme of the previous Sec. IVA. As a result, one can obtain finite and renormalized expression for the TDP at nonzero $T$,

$$
\begin{aligned}
\Omega_{T}^{r e n}\left(M, b, b^{\prime}, \Delta\right) & =\Omega^{r e n}\left(M, b, b^{\prime}, \Delta\right) \\
& -T \int_{0}^{\infty} \frac{d p_{1}}{2 \pi} \ln \left\{\left[1+e^{-\beta\left|P_{01}\right|}\right]\left[1+e^{-\beta\left|P_{02}\right|}\right]\left[1+e^{-\beta\left|\bar{P}_{01}\right|}\right]\left[1+e^{-\beta\left|\bar{P}_{02}\right|}\right]\right\},
\end{aligned}
$$

where $\Omega^{r e n}\left(M, b, b^{\prime}, \Delta\right)$ is the zero temperature TDP (46). Based on the numerical algorithm for finding the quasiparticle energies $P_{01}, P_{02}, \bar{P}_{01}, \bar{P}_{02}$ (see Appendix B), it is possible to show that at fixed values of the variables $\tilde{\mu}$ and $\tilde{\mu}_{5}$ the least value of the TDP (55) can never be achieved at the point $(M, \Delta)$ with both nonzero coordinates, $M \neq 0$ and $\Delta \neq 0$. So to investigate the global minimum of this TDP it is sufficient to deal with the restrictions of the TDP (55) on the manifolds $\Delta=0$ and $M=0$, i.e. with the quantities

$$
\Omega_{1 T}\left(M, b, b^{\prime}\right) \equiv \Omega_{T}^{r e n}\left(M, b, b^{\prime}, \Delta=0\right), \quad \Omega_{2 T}\left(\Delta, b, b^{\prime}\right) \equiv \Omega_{T}^{r e n}\left(M=0, b, b^{\prime}, \Delta\right),
$$


correspondingly. Note that at $\Delta=0$ we have from (16) that each of quasiparticle energies $P_{01}, P_{02}, \bar{P}_{01}$, and $\bar{P}_{02}$ is equal to one of the expressions $\tilde{\mu} \pm \sqrt{M^{2}+\left(p_{1}-\tilde{\mu}_{5}\right)^{2}}$ or $-\tilde{\mu} \pm \sqrt{M^{2}+\left(p_{1}+\tilde{\mu}_{5}\right)^{2}}$, whereas at $M=0$ one can easily see from (17) that each of these quantities is represented by one of the expressions $\tilde{\mu}_{5} \pm \sqrt{\Delta^{2}+\left(p_{1}-\tilde{\mu}\right)^{2}}$ or $-\tilde{\mu}_{5} \pm \sqrt{\Delta^{2}+\left(p_{1}+\tilde{\mu}\right)^{2}}$. Then, one should take into account the expression (46) for the TDP $\Omega^{r e n}\left(M, b, b^{\prime}, \Delta\right)$ as well as the relations (31) and (32) for particular values of the TDP (30) at $\Delta=0$ and $M=0$. Finally, when converting the integral term of (55) we use essentially the following relation

$$
\ln \left(1+e^{-x}\right)=-x+\ln \left(1+e^{x}\right) .
$$

As a result, we obtain the following expressions for the TDPs (56):

$$
\begin{aligned}
\Omega_{1 T}\left(M, b, b^{\prime}\right)= & \frac{M^{2}}{2 \pi} \ln \left(\frac{M}{M_{1}}\right)-\frac{M^{2}}{4 \pi}+\frac{\tilde{\mu}^{2}}{2 \pi}-\frac{\mu^{2}}{2 \pi}-\frac{\mu_{5}^{2}}{2 \pi} \\
& -T \int_{0}^{\infty} \frac{d q}{\pi} \ln \left\{\left[1+e^{-\beta\left(\sqrt{M^{2}+q^{2}}+\tilde{\mu}\right)}\right]\left[1+e^{-\beta\left(\sqrt{M^{2}+q^{2}}-\tilde{\mu}\right)}\right]\right\}, \\
\Omega_{2 T}\left(\Delta, b, b^{\prime}\right)= & \frac{\Delta^{2}}{2 \pi} \ln \left(\frac{\Delta}{M_{2}}\right)-\frac{\Delta^{2}}{4 \pi}+\frac{\tilde{\mu}_{5}^{2}}{2 \pi}-\frac{\mu^{2}}{2 \pi}-\frac{\mu_{5}^{2}}{2 \pi} \\
& -T \int_{0}^{\infty} \frac{d q}{\pi} \ln \left\{\left[1+e^{-\beta\left(\sqrt{\Delta^{2}+q^{2}}+\tilde{\mu}_{5}\right)}\right]\left[1+e^{-\beta\left(\sqrt{\Delta^{2}+q^{2}}-\tilde{\mu}_{5}\right)}\right]\right\} .
\end{aligned}
$$

Note that the function (57) (the function (58)) does not depend on the variable $b^{\prime}$ (variable $b$ ). Due to this fact, it is possible to establish that the TDP (57) has two stationary points, $\left(M=M_{0}(T), b=\mu, b^{\prime}=0\right)$ and $(M=0, b=$ $\left.0, b^{\prime}=0\right)$, where $M_{0}(T)$ vs $T$ behaves like the gap in ordinary Gross-Neveu model with zero chemical potential and $T \neq 0$ [38], i.e. $M_{0}(0)=M_{1}$ and $M_{0}\left(T_{c 1}\right)=0$, where $T_{c 1}=M_{1} e^{\gamma} / \pi$ (here $\gamma$ is the Euler's constant, $\gamma=0.577 \ldots$ ). By analogy, the TDP (58) also has two stationary points, $\left(\Delta=\Delta_{0}(T), b=0, b^{\prime}=\mu_{5}\right)$ and $\left(\Delta=0, b=0, b^{\prime}=0\right)$, with similar properties of the gap $\Delta_{0}(T)$ vs $T: \Delta_{0}(0)=M_{2}$ and $\Delta_{0}\left(T_{c 2}\right)=0$, where $T_{c 2}=M_{2} e^{\gamma} / \pi$. Comparing the values of the TDPs (57) and (58) in the above-mentioned stationary points, it is possible to find the genuine GMP of the initial TDP (55) and, as a consequence, to establish the phase structure of the model at each fixed value of chemical potentials and temperature. It turns out that at $M_{1}>M_{2}$ the inhomogeneous chiral symmetry breaking (or chiral density wave) phase is realized in the model at $T<T_{c 1}$ for arbitrary $\mu>0$ and $\mu_{5}>0$ values. However, at $T>T_{c 1}$ one can observe in this case the symmetrical phase. In contrast, at $M_{1}<M_{2}$ the dual phase portrait is realized in the model: in this case we have an inhomogeneous superconducting phase at $T<T_{c 2}$ and a symmetrical phase at $T>T_{c 2}$. If $M_{1}=M_{2}$, then at $T<T_{c 1}$ there is a degeneracy between inhomogeneous CSB and inhomogeneous SC phases, whereas at $T>T_{c 1}$ the symmetrical phase is realized.

Finally, a few words are in order about the behavior of the particle number $n$ and chiral charge $n_{5}$ densities at nonzero temperature. Recall that to find these quantities we should first of all obtain the value of the TDP (55) in its global minimum point $\left(M_{0}, b_{0}, b_{0}^{\prime}, \Delta_{0}\right)$. Then, particle number density $n$ (chiral charge density $n_{5}$ ) is the derivative of the quantity $\Omega^{r e n}\left(M_{0}, b_{0}, b_{0}^{\prime}, \Delta_{0}\right)$ with respect to chemical potential $\mu$ (chemical potential $\mu_{5}$ ). Hence, taking into account the above consideration of the phase structure, it is possible to conclude that both at $M_{1}>M_{2}$ and $M_{1}<M_{2}$ the same simple expressions for densities,

$$
n=\frac{\mu}{\pi}, \quad n_{5}=\frac{\mu_{5}}{\pi},
$$

are valid for arbitrary temperatures, i.e. in the symmetric phase, in the inhomogeneous chiral symmetry breaking phase (at $T<T_{c 1}$ if $M_{1}>M_{2}$ ) and in the inhomogeneous superconducting phase (at $T<T_{c 2}$ if $M_{1}<M_{2}$ ).

\section{SUMMARY AND DISCUSSIONS}

In this paper, some thermodynamical properties of the (1+1)-dimensional system, which is characterized by ground states with nonzero particle number as well as the chiral charge densities, are considered. The microscopic Lagrangian, describing physics of the system, is chosen in the form (1); i.e. we deal with the (1+1)-dimensional NJL model, containing two types, or channels, of interaction. In the first, chiral, channel the interaction between particles and antiparticles is characterized by coupling constant $G_{1}$, whereas in the second, superconducting channel, we have particle-particle interaction with coupling $G_{2}$. The phase structure of the model is investigated in the paper in terms of particle number $\mu$ and chiral charge $\mu_{5}$ chemical potentials.

There are several reasons for taking into consideration two types of chemical potentials. The first and the most important one is to bring the investigation of the duality between chiral symmetry breaking and superconductivity to a single platform, i.e. to extend the investigation of the duality to the framework of a more general (1+1)-dimensional model (1), rather than the way it was done earlier in [31]. Recall that in [31], a connection was found (duality) between properties of two different models, the GN model with chemical potential $\mu$, describing quark interaction 
in the $q \bar{q}$ channel only, and the GN model with chemical potential $\mu_{5}$, describing the interaction in the $q q$ channel only. In contrast, in our model (1) there is a competition between these two types of interaction and, in addition, there are both types of chemical potentials, $\mu$ and $\mu_{5}$. The second reason is already a "traditional" motivation, which is common in all investigations of low-dimensional theories, i.e. the possibility and the hope to perform a deeper consideration of a new physical phenomena in terms of toy models. In our case these are the parity breaking [23, 24] and the chiral magnetic [25] effects of dense quark-gluon matter, accompanied by a nonzero chiral charge density $n_{5}$ $\left(\right.$ or $\left.\mu_{5} \neq 0\right)$.

Moreover, the finite temperature effect is also taken into account. It is well known that in any dense system there might appear a spontaneous breaking of spatial translational invariance, resulting in a spatial dependence of order parameters, or condensates. So we investigated a phase structure of the model, assuming the Fulde-Ferrel [22] single plane wave ansatz (10) for condensates. (In particular, for the chiral condensate the ansatz (10) is known as a chiral density wave or chiral spiral.) For comparison, we investigate a phase structure of the model in two particular cases of the ansatz (10): (i) when $b=0$ and $b^{\prime}=0$, i.e. the condensates are put as spatially homogeneous by hand, and (ii) when the parameters $b, b^{\prime}$ are dynamical quantities, defined by gap equations. The main results of the paper are the following.

(1) First of all, we have established that in the homogeneous case of the ansatz (10) for condensates (at $b=0$ and $\left.b^{\prime}=0\right)$ the thermodynamic potential of the model is invariant under the duality transformation $D$ (18). It means that if at $G_{1}>G_{2}$ (or, equivalently, at $M_{1}>M_{2}$, where the connections between $G_{1,2}$ and $M_{1,2}$ are represented in (21)) the CSB phase (SC phase) is realized in the model at some fixed values of chemical potentials, e.g., at $\mu=\alpha$, $\mu_{5}=\beta$, then at $G_{1} \leftrightarrow G_{2}$ the system is in the SC phase (CSB phase) at $\mu=\beta, \mu_{5}=\alpha$. Taking into account this duality correspondence property of the model, it is sufficient to study the $\left(\mu, \mu_{5}\right)$-phase diagram only at $G_{1}>G_{2}$, i.e. at $M_{1}>M_{2}$ (see, e.g., Fig. 1). Then the phase portrait of the model at $M_{1}<M_{2}$ (see Fig. 2) is simply the dual mapping of Fig. 1.

(2) At $G_{1}=G_{2}$ (or at $\left.M_{1}=M_{2}\right)$ the $\left(\mu, \mu_{5}\right)$-phase diagram of the model in the homogeneous case of the ansatz (10) for condensates is presented in Fig. 3. Clearly, this diagram is invariant with respect to the duality transformation $D$ (18); i.e. one can say that the model is self-dual in this case. Nevertheless, we would like to emphasize that in the homogeneous case of the ansatz (10) and at $\mu \neq \mu_{5}$ the self-duality property of the model does not mean the degeneracy of the CSB and SC ground states at $G_{1}=G_{2}$. The CSB-SC degeneracy appears only on the line L of Fig. 3 , i.e. at $\mu=\mu_{5}$.

(3) If a spatially inhomogeneous behavior of condensates is assumed in the form (10), where the parameters $b$ and $b^{\prime}$ must be found by gap equations, then the $\left(\mu, \mu_{5}\right)$-phase structure of the model is considerably simplified. Indeed, in this case at $G_{1}>G_{2}$, i.e. at $M_{1}>M_{2}$, (at $G_{1}<G_{2}$ ) only the inhomogeneous chiral density wave phase (only inhomogeneous SC phase) is realized in the model for arbitrary values of $\mu$ and $\mu_{5}$. The critical temperature, at which the inhomogeneous chiral density wave phase (the inhomogeneous SC phase) is destroyed and the symmetrical phase appears, is equal to $T_{c 1}=M_{1} e^{\gamma} / \pi$ (equal to $T_{c 2}=M_{2} e^{\gamma} / \pi$ ). (In contrast, if $b$ and $b^{\prime}$ are equal to zero a priori, i.e. condensates are assumed to be homogeneous from the very beginning, then, depending on the relation between $\mu$ and $\mu_{5}$, spatially uniform CSB and SC phases are present on a model phase portrait both at $G_{1}>G_{2}$ and $G_{1}<G_{2}$ (see, e.g., Figs 1,2).) Note also that if $G_{1} \neq G_{2}$, then the inhomogeneous chiral density wave phase is a dual mapping of the inhomogeneous SC phase and vice versa. Moreover, in this case the degeneracy between the above-mentioned inhomogeneous phases is absent.

(4) It is interesting to note that at $G_{1}=G_{2}$ and for arbitrary fixed values of the chemical potentials $\mu$ and $\mu_{5}$ the self-dual and degenerated phase portrait of the above-mentioned inhomogeneous phases appears. It means that for each fixed value of $\mu$ and $\mu_{5}$ there is an equal opportunity for the emergence as one or the other inhomogeneous phase in the system. Moreover, the coexistence of these phases is not excluded. In contrast, in the homogeneous case of the ansatz (10) for condensates the degeneration between CSB and SC ground states of the model is absent (at $\left.\mu \neq \mu_{5}\right)$, in spite of a self-dual phase portrait of the model at $G_{1}=G_{2}$ (see Fig. 3).

(5) Note that if the condesates are homogeneous and $T=0$, then in the CSB phase the particle density $n$ is identically zero, whereas the chiral charge density $n_{5}$ vanishes in the SC phase (see (38) and (39)). However, if a possibility of spatial inhomogeneity for condensates in the form (10) is taken into account, then both in the ground state corresponding to an inhomogeneous chiral density wave phase and in the inhomogeneous SC phase the nonzero particle number density, $n=\mu / \pi$, and nonzero chiral charge density, $n_{5}=\mu_{5} / \pi$, appear. Moreover, at $T \neq 0$ and in the case of inhomogeneous ansatz (10) for condensates both particle density $n$ and chiral charge density $n_{5}$ do not depend on temperature and have the same behaviors (59) in all possible phases of the model, symmetrical, inhomogeneous chiral density wave, and inhomogeneous superconducting phases.

We are aware of the fact that some of the above properties (such as the appearance of an inhomogeneous phase at arbitrarily low chemical potentials or a change of the nature of the inhomogeneous phase (CSB vs SC) if the SC coupling becomes larger than the CSB one) are peculiarities of the above-considered (1+1)-dimensional model (1) and, perhaps, have no relation to reality. However, there are results (e.g. the extension of inhomogeneous phases to high values of chemical potentials) that are predicted by $(3+1)$-dimensional QCD-like models as well [13].

It is also worth noting that in the recent paper [24] the $(3+1)$-dimensional NJL model with several quark-antiquark interaction channels was investigated at zero temperature and in the presence of two chemical potentials, $\mu$ and $\mu_{5}$. 
The only homogeneous ansatz for condensates is taken into account in this research, which is devoted to the study of the parity breaking effects in QCD at high densities. As it was established there, in dense quark matter, i.e. at $\mu \neq 0$, and at a rather high values of $\mu_{5}$ the chiral symmetry breaking + parity breaking phase is allowed to exist (see Fig. 7 in this paper). In some ways our work is related to the same problem, but only considered in the framework of a simple $\mathrm{NJL}_{2}$ toy model (1). Indeed, we have shown that at $T<T_{c 1}$ and $G_{1}>G_{2}$ an inhomogeneous CSB phase, in which parity is also spontaneously broken down, is realized in the model (1). So, there is an alternative mechanism to achieve parity breaking in dense QCD, based on spatially nonuniform condensates. 10 Moreover, we have demonstrated that, in comparison with the NJL model [24], a reduced number of quark-antiquark channels of interaction is needed in order to obtain spontaneous parity and chiral symmetry breaking at $\mu \neq 0$.

Finally, note that inhomogeneous phases are observed in a phase diagram of the $\mathrm{NJL}_{2}$ model (1) only at small temperatures, i.e. at $T<T_{c 1}$ if $G_{1}>G_{2}$ or at $T<T_{c 2}$ if $G_{1}<G_{2}$. At high temperatures the symmetric phase with $n \neq 0$ and, especially, with nonzero chiral charge density $n_{5}$ is realized (see in Sec. IV C). It is well known that in a heavy ion collision scenario both the temperature and magnetic field can be extremely high. Due to high temperatures a sphaleron transition might occur, which is accompanied by an appearance of a nonzero chiral density $n_{5}$ (or chemical potential $\mu_{5} \neq 0$ ) in the system $[25,26]$. Due to strong magnetic fields the dynamics of the system becomes essentially one dimensional. So we believe that in the high temperature region the model (1) reflects in some details the physics of quark-gluon plasma and, furthermore, the (1+1)-dimensional models with $\mu_{5}$-chemical potential deserve to be investigated.

\section{Appendix A: The path integration over anticommuting fields}

Let us calculate the following path integral over anticommuting two-component Dirac spinor fields $q(x), \bar{q}(x)$ :

$$
I=\int[d \bar{q}][d q] \exp \left(i \int d^{2} x\left[\bar{q} D q-\frac{\Delta}{2}\left(q^{T} \epsilon q\right)-\frac{\Delta}{2}\left(\bar{q} \epsilon \bar{q}^{T}\right)\right]\right),
$$

where we use the notations of Sec. III. In particular, the operator $D$ is given in (13) and $\epsilon$ is defined in (2). Note that the integral $I$ is equal to the argument of the $\ln (x)$ function in the formula (12) in the particular case $N=1$. Recall that there are general Gaussian path integrals [39]:

$$
\begin{aligned}
& \int[d q] \exp \left(i \int d^{2} x\left[-\frac{1}{2} q^{T} A q+\eta^{T} q\right]\right)=(\operatorname{det}(A))^{1 / 2} \exp \left(-\frac{i}{2} \int d^{2} x\left[\eta^{T} A^{-1} \eta\right]\right), \\
& \int[d \bar{q}] \exp \left(i \int d^{2} x\left[-\frac{1}{2} \bar{q} A \bar{q}^{T}+\bar{\eta} \bar{q}^{T}\right]\right)=(\operatorname{det}(A))^{1 / 2} \exp \left(-\frac{i}{2} \int d^{2} x\left[\bar{\eta} A^{-1} \bar{\eta}^{T}\right]\right),
\end{aligned}
$$

where $A$ is an antisymmetric operator in coordinate and spinor spaces, and $\eta(x), \bar{\eta}(x)$ are spinor anticommuting sources which also anticommute with $q$ and $\bar{q}$. First, let us integrate in (A1) over $q$ fields with the help of the relation (A2) supposing there that $A=\Delta \epsilon, \bar{q} D=\eta^{T}$, i.e. $\eta=D^{T} \bar{q}^{T}$. Then

$$
I=(\operatorname{det}(\Delta \epsilon))^{1 / 2} \int[d \bar{q}] \exp \left(-\frac{i}{2} \int d^{2} x \bar{q}\left[\Delta \epsilon+D(\Delta \epsilon)^{-1} D^{T}\right] \bar{q}^{T}\right) .
$$

Second, the integration over $\bar{q}$ fields in (A4) can be easily performed with the help of the formula (A3), where one should put $A=\Delta \epsilon+D(\Delta \epsilon)^{-1} D^{T}$ and $\bar{\eta}=0$. As a result, we have

$$
I=(\operatorname{det}(\Delta \epsilon))^{1 / 2}\left(\operatorname{det}\left[\Delta \epsilon+D(\Delta \epsilon)^{-1} D^{T}\right]\right)^{1 / 2}=\left(\operatorname{det}\left[-\Delta^{2}-D \epsilon D^{T} \epsilon\right]\right)^{1 / 2},
$$

where we took into account that $\epsilon \epsilon=-1$ and $\epsilon^{-1}=-\epsilon$. For the following one should remember the well-known relations: $\left(\partial_{\nu}\right)^{T}=-\partial_{\nu}, \epsilon\left(\gamma^{\nu}\right)^{T} \epsilon=\gamma^{\nu}$, where $\nu=0,1$. Hence,

$$
I=\left(\operatorname{det}\left[-\Delta^{2}+D_{+} D_{-}\right]\right)^{1 / 2} \equiv(\operatorname{det} B)^{1 / 2},
$$

where $D_{ \pm}=\gamma^{\nu} i \partial_{\nu}-M \pm\left((\mu-b) \gamma^{0}+\left(\mu_{5}-b^{\prime}\right) \gamma^{1}\right)$. Using the general relation $\operatorname{det} B=\exp (\operatorname{Tr} \ln B)$, we get from (A6):

$$
\ln I=\frac{1}{2} \operatorname{Tr} \ln \left(-\Delta^{2}+D_{+} D_{-}\right)=\sum_{i=1}^{2} \int \frac{d^{2} p}{(2 \pi)^{2}} \ln \left(\lambda_{i}(p)\right) \int d^{2} x
$$

10 As it follows from our consideration, the role of the chiral chemical potential $\mu_{5}$ in this approach is simply to supply a nonzero chiral charge density $n_{5}=\mu_{5} / \pi$ (59) in the chiral density wave phase, where both parity and chiral symmetry are broken spontaneously. 
(A more detailed consideration of operator traces is presented in Appendix A of the paper [40].) In this formula the symbol Tr means the trace of an operator both in the coordinate and internal spaces. Moreover, $\lambda_{i}(p)(i=1,2)$ are eigenvalues of the $2 \times 2$ Fourier transformed matrix $\bar{B}(p)$ of the operator $B$, i.e.

$$
\lambda_{1,2}(p)=p_{0}^{2}-\tilde{\mu}^{2}-p_{1}^{2}+\tilde{\mu}_{5}^{2}+M^{2}-\Delta^{2} \pm 2 \sqrt{M^{2} p_{0}^{2}-M^{2} p_{1}^{2}+\tilde{\mu}^{2} p_{1}^{2}-2 p_{0} \tilde{\mu}_{5} \tilde{\mu} p_{1}+p_{0}^{2} \tilde{\mu}_{5}^{2}},
$$

where $\tilde{\mu}=\mu-b$ and $\tilde{\mu}_{5}=\mu_{5}-b^{\prime}$.

\section{Appendix B: Evaluation of the TDP (15)}

In order to renormalize and then to investigate the TDP (15), it is necessary to modify the initial expression (15). First let us obtain a more convenient expression for $\operatorname{det} \bar{B}(p)$. With this aim we use the following alternative form of the relation (16), namely

$$
\begin{aligned}
\operatorname{det} \bar{B}(p) & =p_{0}^{4}-2\left(M^{2}+\Delta^{2}+\mu^{2}+\mu_{5}^{2}+p_{1}^{2}\right) p_{0}^{2}+8 \mu_{5} \mu p_{1} p_{0}+p_{1}^{4}-2 p_{1}^{2}\left(\mu^{2}+\mu_{5}^{2}-M^{2}-\Delta^{2}\right) \\
& +\left(\Delta^{2}-M^{2}+\mu^{2}-\mu_{5}^{2}\right)^{2} \equiv p_{0}^{4}+\alpha p_{0}^{2}+\beta p_{0}+\gamma,
\end{aligned}
$$

where, evidently,

$$
\begin{aligned}
& \alpha=-2\left(M^{2}+\Delta^{2}+\mu^{2}+\mu_{5}^{2}+p_{1}^{2}\right), \quad \beta=8 \mu_{5} \mu p_{1}, \\
& \gamma=p_{1}^{4}-2 p_{1}^{2}\left(\mu^{2}+\mu_{5}^{2}-M^{2}-\Delta^{2}\right)+\left(\Delta^{2}-M^{2}+\mu^{2}-\mu_{5}^{2}\right)^{2} .
\end{aligned}
$$

It is very convenient to present the fourth-order polynomial of the variable $p_{0}(\mathrm{~B} 1)$ as a product of two second-order polynomials (this way is proposed in [41]); i.e. we assume that

$$
\begin{aligned}
p_{0}^{4}+ & \alpha p_{0}^{2}+\beta p_{0}+\gamma=\left(p_{0}^{2}+r p_{0}+q\right)\left(p_{0}^{2}-r p_{0}+s\right) \\
& =\left[\left(p_{0}+\frac{r}{2}\right)^{2}+q-\frac{r^{2}}{4}\right]\left[\left(p_{0}-\frac{r}{2}\right)^{2}+s-\frac{r^{2}}{4}\right] \equiv\left(p_{0}-p_{01}\right)\left(p_{0}-p_{02}\right)\left(p_{0}-\bar{p}_{01}\right)\left(p_{0}-\bar{p}_{02}\right),
\end{aligned}
$$

where $r, q$ and $s$ are some real valued quantities, such that

$$
\alpha=s+q-r^{2}, \quad \beta=r s-q r, \quad \gamma=s q .
$$

Then, using expansion (B3), it is easy to present all the roots $p_{01}, p_{02}, \bar{p}_{01}$, and $\bar{p}_{02}$ of the polynomial (B1)-(B3) vs $p_{0}$ in the following form:

$$
p_{01}=-\frac{r}{2}+\sqrt{\frac{r^{2}}{4}-q}, \quad p_{02}=\frac{r}{2}+\sqrt{\frac{r^{2}}{4}-s}, \quad \bar{p}_{01}=-\frac{r}{2}-\sqrt{\frac{r^{2}}{4}-q}, \quad \bar{p}_{02}=\frac{r}{2}-\sqrt{\frac{r^{2}}{4}-s} .
$$

The expressions (B5) are usually called the dispersion laws (or relations) of the model. So, the quantities $p_{01}$ and $p_{02}$ are the energies of two quasiparticles, whereas $\bar{p}_{01}$ and $\bar{p}_{02}$ are the energies of their quasiantiparticles. Since in (B3) the energy parameter $p_{0}$ is shifted by $\pm r / 2$, one may interpret the quantity $r / 2$ as an effective chemical potential. In the following we are going to use just the quantities (B5) in our numerical calculations, so it is necessary to express the coefficients $r, q$ and $s$ in (B3) in terms of $\alpha, \beta, \gamma$-quantities.

Suppose first that $\mu=0$ and $\mu_{5}=0$ (other variables, $M, \Delta$, and $p_{1}$, are nonzero). Then, as it is clear from (19), $r=0, s=-p_{1}^{2}-(M-\Delta)^{2}$ and $q=-p_{1}^{2}-(M+\Delta)^{2}$. In particular, it means that in this case

$$
\left.\left(\left|p_{01}\right|+\left|p_{02}\right|+\left|\bar{p}_{01}\right|+\left|\bar{p}_{02}\right|\right)\right|_{\mu=0, \mu_{5}=0}=2 \sqrt{p_{1}^{2}+(M+\Delta)^{2}}+2 \sqrt{p_{1}^{2}+(M-\Delta)^{2}} .
$$

In the general case, when both $\mu \neq 0$ and $\mu_{5} \neq 0$, one can solve the system of equations (B4) and find

$$
q=\frac{1}{2}\left(\alpha+R-\frac{\beta}{\sqrt{R}}\right), \quad s=\frac{1}{2}\left(\alpha+R+\frac{\beta}{\sqrt{R}}\right), \quad r=\sqrt{R},
$$

where $R$ is an arbitrary positive real solution of the equation

$$
X^{3}+A X=B X^{2}+C
$$

with respect to a variable $X$, and

$$
\begin{aligned}
& A=\alpha^{2}-4 \gamma=16\left[\mu_{5}^{2} \Delta^{2}+M^{2} \mu^{2}+\Delta^{2} M^{2}+\mu_{5}^{2} \mu^{2}+p_{1}^{2}\left(\mu^{2}+\mu_{5}^{2}\right)\right], \\
& B=-2 \alpha=4\left(M^{2}+\Delta^{2}+\mu^{2}+\mu_{5}^{2}+p_{1}^{2}\right), \quad C=\beta^{2}=\left(8 \mu_{5} \mu p_{1}\right)^{2} .
\end{aligned}
$$

Numerical investigation shows that for any fixed values of $\mu>0, \mu_{5}>0, M>0, \Delta>0$ and $p_{1}$ the discriminant of the third-order algebraic equation (B8), i.e. the quantity $18 A B C-4 B^{3} C+A^{2} B^{2}-4 A^{3}-27 C^{2}$, is always positive. 
So the equation (B8) vs $X$ has three different real solutions $R_{1}, R_{2}$ and $R_{3}$ (this fact is presented in [41]). Moreover, since the coefficients $A, B$ and $C$ (B9) are positively defined, it is clear that all the roots $R_{1}, R_{2}$ and $R_{3}$ are positive quantities. So we are free to choose the quantity $R$ from (B7) as one of the solutions $R_{1}, R_{2}$ or $R_{3}$. In each case, i.e. for $R=R_{1}, R=R_{2}$, or $R=R_{3}$, we will obtain the same set of the roots (B5) (possibly rearranged), which depends only on $\mu, \mu_{5}, M, \Delta$ and $p_{1}$, and does not depend on the choice of $R$.

Using standard methods, it is possible to find the following $p_{1} \rightarrow \infty$ asymptotic expansions of the roots $R_{1}, R_{2}$ and $R_{3}$,

$$
\begin{aligned}
& R_{1}=4 \mu^{2}+\frac{4 \Delta^{2} \mu^{2}\left[\mu^{2}-M^{2}-\mu_{5}^{2}\right]}{\left(\mu_{5}^{2}-\mu^{2}\right) p_{1}^{2}}+\mathcal{O}\left(1 / p_{1}^{4}\right), \\
& R_{2}=4 \mu_{5}^{2}+\frac{4 M^{2} \mu_{5}^{2}\left[\mu_{5}^{2}-\Delta^{2}-\mu^{2}\right]}{\left(\mu^{2}-\mu_{5}^{2}\right) p_{1}^{2}}+\mathcal{O}\left(1 / p_{1}^{4}\right), \\
& R_{3}=4 p_{1}^{2}+4\left(M^{2}+\Delta^{2}\right)+\frac{4\left(\mu_{5}^{2} M^{2}+\mu^{2} \Delta^{2}-M^{2} \Delta^{2}\right)}{p_{1}^{2}}+\mathcal{O}\left(1 / p_{1}^{4}\right) .
\end{aligned}
$$

It is clear from these relations that $R_{3}$ is invariant, whereas $R_{1} \leftrightarrow R_{2}$ under the duality transformation (18). Note that the expansions (B10) and (B11) are valid only at $\mu_{5} \neq \mu$. If $\mu_{5}=\mu$, then at $p_{1} \rightarrow \infty$ we have for $R_{1,2}$ the expansions

$$
R_{1,2}=4 \mu^{2} \pm \frac{4 \mu \Delta M}{\left|p_{1}\right|}+\frac{2 \Delta^{2} M^{2}-2 \mu^{2} \Delta^{2}-2 \mu^{2} M^{2}}{p_{1}^{2}}+\mathcal{O}\left(1 / p_{1}^{3}\right) .
$$

(In this particular case each of the roots $R_{1,2,3}$ is invariant with respect to the duality transformation (18).) It was mentioned above that the quantity $r / 2$ can be interpreted as an effective chemical potential (see the text after (B5)). Moreover, it is clear from (B10) that just the choice $R=R_{1}$ supports this interpretation, since at $p_{1} \rightarrow \infty$ we have $r / 2=\sqrt{R_{1}} / 2 \rightarrow \mu$. Besides, if the quantity $R$ from (B7) is equal to the root $R_{1}$, then it is easy to obtain asymptotic expansions at $p_{1} \rightarrow \infty$ of quasiparticle energies,

$$
\begin{aligned}
& \left|p_{01}\right|=\left|p_{1}\right|-\mu+\mu_{5}+\frac{\Delta^{2}+M^{2}}{2\left|p_{1}\right|}+\mathcal{O}\left(1 / p_{1}^{2}\right), \quad\left|\bar{p}_{01}\right|=\left|p_{1}\right|+\mu+\mu_{5}+\frac{\Delta^{2}+M^{2}}{2\left|p_{1}\right|}+\mathcal{O}\left(1 / p_{1}^{2}\right), \\
& \left|p_{02}\right|=\left|p_{1}\right|+\mu-\mu_{5}+\frac{\Delta^{2}+M^{2}}{2\left|p_{1}\right|}+\mathcal{O}\left(1 / p_{1}^{2}\right), \quad\left|\bar{p}_{02}\right|=\left|p_{1}\right|-\mu-\mu_{5}+\frac{\Delta^{2}+M^{2}}{2\left|p_{1}\right|}+\mathcal{O}\left(1 / p_{1}^{2}\right),
\end{aligned}
$$

which follow from (B10)-(B12) as well as from the relations (B7) and (B5). As a result, it can be established from (B14) the asymptotic expansion (26). We would like to emphasize once again that the asymptotic behavior (26) does not depend on which of the roots $R_{1}, R_{2}$ or $R_{3}$ of the equation (B8) is taken as the quantity $R$ from the relations (B7).

Now, taking into account the relations (B1) and (B3) one can integrate over $p_{0}$ in (15), using the well-known formula

$$
\int_{-\infty}^{\infty} d p_{0} \ln \left(p_{0}-A\right)=i \pi|A|
$$

(obtained rigorously, e.g., in Appendix B of [20] and true up to an infinite term independent on the real quantity $A$ ). As a result, we have

$$
\begin{aligned}
\frac{i}{2} \int \frac{d^{2} p}{(2 \pi)^{2}} \ln [\operatorname{det} \bar{B}(p)] & =-\int_{-\infty}^{\infty} \frac{d p_{1}}{8 \pi}\left\{\left|p_{01}\right|+\left|p_{02}\right|+\left|\bar{p}_{01}\right|+\left|\bar{p}_{02}\right|\right\} \\
& =-\int_{0}^{\infty} \frac{d p_{1}}{4 \pi}\left\{\left|p_{01}\right|+\left|p_{02}\right|+\left|\bar{p}_{01}\right|+\left|\bar{p}_{02}\right|\right\},
\end{aligned}
$$

where the expressions for energies of quasiparticles, $p_{01}$ and $p_{02}$, as well as for energies of quasiantiparticles, $\bar{p}_{01}$ and $\bar{p}_{02}$, are given in (B5). Note that the last equality in (B16) is due to the fact that the sum $\left(\left|p_{01}\right|+\left|p_{02}\right|+\left|\bar{p}_{01}\right|+\left|\bar{p}_{02}\right|\right)$ is an even function of $p_{1}$, as it is easily seen from (B5) and (B7). Moreover, due to (B5) one can obtain an equivalent expression for (B16),

$$
\begin{aligned}
\frac{i}{2} \int \frac{d^{2} p}{(2 \pi)^{2}} \ln [\operatorname{det} \bar{B}(p)] & =-\int_{0}^{\infty} \frac{d p_{1}}{4 \pi}\left\{\sqrt{r^{2}-4 q}+\sqrt{r^{2}-4 s}\right. \\
& \left.+\left(r-\sqrt{r^{2}-4 q}\right) \theta\left(r-\sqrt{r^{2}-4 q}\right)+\left(r-\sqrt{r^{2}-4 s}\right) \theta\left(r-\sqrt{r^{2}-4 s}\right)\right\},
\end{aligned}
$$

where $r, q$ and $s$ are defined in $(\overline{\mathrm{B} 3})-(\mathrm{B} 7)$ and $\theta(x)$ is the Heaviside step function. It is very convenient to use this relation in the cases $r=\sqrt{R_{1}}$ and $r=\sqrt{R_{2}}$. In these cases an ultraviolet divergence of the integral (B17) is due to the first two terms in the braces, whereas the terms with $\theta(x)$ functions do not generate any divergences there. 
Finally, in addition to (B6), we would like to present two other particular cases, in which the expressions for the quasiparticle energies (B5) and the sum $\left(\left|p_{01}\right|+\left|p_{02}\right|+\left|\bar{p}_{01}\right|+\left|\bar{p}_{02}\right|\right)$ can be given in an exact analytical form. Namely, if $\Delta=0$ then we have from (16) that each of $p_{01}, p_{02}, \bar{p}_{01}$, and $\bar{p}_{02}$ is given by one of the expressions $\mu \pm \sqrt{M^{2}+\left(p_{1}-\mu_{5}\right)^{2}}$ or $-\mu \pm \sqrt{M^{2}+\left(p_{1}+\mu_{5}\right)^{2}}$. Therefore, their sum is represented by

$$
\begin{aligned}
\left.\left(\left|p_{01}\right|+\left|p_{02}\right|+\left|\bar{p}_{01}\right|+\left|\bar{p}_{02}\right|\right)\right|_{\Delta=0} & = \\
& \sum_{\eta= \pm}\left(\left|\mu+\eta \sqrt{M^{2}+\left(p_{1}-\eta \mu_{5}\right)^{2}}\right|+\left|\mu+\eta \sqrt{M^{2}+\left(p_{1}+\eta \mu_{5}\right)^{2}}\right|\right) .
\end{aligned}
$$

Analogously, at $M=0$ we have from (17)

$$
\begin{aligned}
\left.\left(\left|p_{01}\right|+\left|p_{02}\right|+\left|\bar{p}_{01}\right|+\left|\bar{p}_{02}\right|\right)\right|_{M=0} & = \\
& \sum_{\eta= \pm}\left(\left|\mu_{5}+\eta \sqrt{\Delta^{2}+\left(p_{1}-\eta \mu\right)^{2}}\right|+\left|\mu_{5}+\eta \sqrt{\Delta^{2}+\left(p_{1}+\eta \mu\right)^{2}}\right|\right) .
\end{aligned}
$$

\section{Appendix C: Derivation of the relation (31)}

If $\Delta=0$ and $M \neq 0$, then the quasiparticle energies (B5) can be easily found from the expression (16). In this case each of $p_{01}, p_{02}, \bar{p}_{01}$, and $\bar{p}_{02}$ is given by one of the expressions $\mu \pm \sqrt{M^{2}+\left(p_{1}-\mu_{5}\right)^{2}}$ or $-\mu \pm \sqrt{M^{2}+\left(p_{1}+\mu_{5}\right)^{2}}$; therefore their sum is represented by the formula (B18). Taking this relation into account as well as using the wellknown relations $|x|=x \theta(x)-x \theta(-x)$ and $\theta(x)=1-\theta(-x)$, it is possible to bring the expression (30) at $\Delta=0$ and $M \neq 0$ to the following form:

$$
\Omega^{r e n}(M, \Delta=0)=-\frac{M^{2}}{4 \pi}+\frac{M^{2}}{2 \pi} \ln \left(\frac{M}{M_{1}}\right)-U-V
$$

where

$$
\begin{gathered}
U=\int_{0}^{\infty} \frac{d p_{1}}{2 \pi}\left\{\sqrt{M^{2}+\left(p_{1}+\mu_{5}\right)^{2}}+\sqrt{M^{2}+\left(p_{1}-\mu_{5}\right)^{2}}-2 \sqrt{M^{2}+p_{1}^{2}}\right\} \\
V=\int_{0}^{\infty} \frac{d p_{1}}{2 \pi}\left\{\left(\mu-\sqrt{M^{2}+\left(p_{1}-\mu_{5}\right)^{2}}\right) \theta\left(\mu-\sqrt{M^{2}+\left(p_{1}-\mu_{5}\right)^{2}}\right)\right. \\
\left.+\left(\mu-\sqrt{M^{2}+\left(p_{1}+\mu_{5}\right)^{2}}\right) \theta\left(\mu-\sqrt{M^{2}+\left(p_{1}+\mu_{5}\right)^{2}}\right)\right\} .
\end{gathered}
$$

The convergent improper integral $U$ can be represented in the form

$$
U=\lim _{\Lambda \rightarrow \infty}\left\{\int_{0}^{\Lambda} \frac{d p_{1}}{2 \pi} \sqrt{M^{2}+\left(p_{1}+\mu_{5}\right)^{2}}+\int_{0}^{\Lambda} \frac{d p_{1}}{2 \pi} \sqrt{M^{2}+\left(p_{1}-\mu_{5}\right)^{2}}-2 \int_{0}^{\Lambda} \frac{d p_{1}}{2 \pi} \sqrt{M^{2}+p_{1}^{2}}\right\}
$$

Denoting the first (second) integral in the braces of (C4) as $U_{1}$ (as $U_{2}$ ) and carrying out there the change of variables, $q=p_{1}+\mu_{5}\left(q=p_{1}-\mu_{5}\right)$, one can obtain

$$
\begin{aligned}
& U_{1}=\int_{\mu_{5}}^{\Lambda+\mu_{5}} \frac{d q}{2 \pi} \sqrt{M^{2}+q^{2}} \equiv\left(\int_{0}^{\Lambda}+\int_{\mu_{5}}^{0}+\int_{\Lambda}^{\Lambda+\mu_{5}}\right) \frac{d q}{2 \pi} \sqrt{M^{2}+q^{2}}, \\
& U_{2}=\int_{-\mu_{5}}^{\Lambda-\mu_{5}} \frac{d q}{2 \pi} \sqrt{M^{2}+q^{2}} \equiv\left(\int_{0}^{\Lambda}+\int_{-\mu_{5}}^{0}+\int_{\Lambda}^{\Lambda-\mu_{5}}\right) \frac{d q}{2 \pi} \sqrt{M^{2}+q^{2}} .
\end{aligned}
$$

Substituting (C5) and (C6) in (C4) and taking into account that

$$
\left(\int_{\mu_{5}}^{0}+\int_{-\mu_{5}}^{0}\right) \frac{d q}{2 \pi} \sqrt{M^{2}+q^{2}}=0
$$

we have after a direct integration

$$
U=\lim _{\Lambda \rightarrow \infty}\left(\int_{\Lambda}^{\Lambda+\mu_{5}}-\int_{\Lambda-\mu_{5}}^{\Lambda}\right) \frac{d q}{2 \pi} \sqrt{M^{2}+q^{2}}=\frac{\mu_{5}^{2}}{2 \pi} .
$$


Analogously, the quantity $V$ (C3) can be represented as a sum of two integrals, in which one should perform a change of variables similar to $U_{1}$ and $U_{2}$, correspondingly. As a result, we have

$$
\begin{aligned}
V & =\int_{-\mu_{5}}^{\infty} \frac{d q}{2 \pi}\left(\mu-\sqrt{M^{2}+q^{2}}\right) \theta\left(\mu-\sqrt{M^{2}+q^{2}}\right)+\int_{\mu_{5}}^{\infty} \frac{d q}{2 \pi}\left(\mu-\sqrt{M^{2}+q^{2}}\right) \theta\left(\mu-\sqrt{M^{2}+q^{2}}\right) \\
& =\theta(\mu-M) \int_{0}^{\sqrt{\mu^{2}-M^{2}}} \frac{d q}{\pi}\left(\mu-\sqrt{M^{2}+q^{2}}\right) .
\end{aligned}
$$

Direct integration in (C9) gives us the following expression for $V$ :

$$
V=\frac{\theta(\mu-M)}{2 \pi}\left(\mu \sqrt{\mu^{2}-M^{2}}-M^{2} \ln \frac{\mu+\sqrt{\mu^{2}-M^{2}}}{M}\right) .
$$

Hence, taking into account the relations (C10), (C8), and (C1), we are convinced of the validity of the formula (31). By analogy, one can derive the expression (32).

\section{Appendix D: Summation over Matsubara frequencies}

Let us sum the series

$$
S(a)=\sum_{n=-\infty}^{\infty} \ln \left(i \omega_{n}-a\right),
$$

where $\omega_{n}=\pi T(2 n+1)$ and $a, T$ are some real quantities. The expression can be modified in the following way:

$$
S(a)=\sum_{n=0}^{\infty}\left\{\ln \left(i \omega_{n}-a\right)+\ln \left(-i \omega_{n}-a\right)\right\}=\sum_{n=0}^{\infty} \ln \left(a^{2}+\omega_{n}^{2}\right) .
$$

It is easy to find from (D2)

$$
\frac{d S(a)}{d a}=2 a \sum_{n=0}^{\infty}\left(a^{2}+\omega_{n}^{2}\right)^{-1}=\frac{\beta}{2} \tanh \left(\frac{\beta a}{2}\right)
$$

where we have used the well-known relation

$$
\sum_{n=0}^{\infty}\left(b^{2}+(2 n+1)^{2}\right)^{-1}=\frac{\pi}{4 b} \tanh \left(\frac{\pi b}{2}\right) .
$$

Finally, integrating both sides of the relation (D3) with respect to the variable $a$ and omitting an unessential constant independent on the quantity $a$, one can obtain

$$
S(a)=\ln [\exp (\beta a / 2)+\exp (-\beta a / 2)]=\ln [\exp (\beta|a| / 2)+\exp (-\beta|a| / 2)]=\frac{\beta|a|}{2}+\ln [1+\exp (-\beta|a|)] .
$$

[1] D.V. Deryagin, D.Y. Grigoriev and V.A. Rubakov, Int. J. Mod. Phys. A 7, 659 (1992); M. Sadzikowski and W. Broniowski, Phys. Lett. B 488, 63 (2000); W. Broniowski, Acta Phys. Polon. Supp. 5, 631 (2012).

[2] E. Nakano and T. Tatsumi, Phys. Rev. D 71, 114006 (2005).

[3] T. Tatsumi and T. Muto, arXiv:1403.1927 [nucl-th]; T. Tatsumi, K. Nishiyama and S. Karasawa, arXiv:1405.2155 [hep-ph].

[4] J. Moreira, B. Hiller, W. Broniowski, A.A. Osipov and A.H. Blin, arXiv:1312.4942 [hep-ph].

[5] D. Nickel, Phys. Rev. D 80, 074025 (2009); S. Carignano, D. Nickel and M. Buballa, Phys. Rev. D 82, 054009 (2010); H. Abuki, D. Ishibashi and K. Suzuki, arXiv:1109.1615.

[6] S. Maedan, Prog. Theor. Phys. 123, 285 (2010); A. Flachi, JHEP 1201, 023 (2012); arXiv:1304.6880 [hep-th].

[7] A. Heinz, arXiv:1301.3430 [hep-ph].

[8] T. Kojo, Y. Hidaka, L. McLerran and R.D. Pisarski, Nucl. Phys. A 843, 37 (2010).

[9] E.V. Gorbar, M. Hashimoto and V.A. Miransky, Phys. Rev. Lett. 96, 022005 (2006); J.O. Andersen and T. Brauner, Phys. Rev. D 81, 096004 (2010); C.f. Mu, L.y. He and Y.x. Liu, Phys. Rev. D 82, 056006 (2010).

[10] I.E. Frolov, K.G. Klimenko and V.Ch. Zhukovsky, Phys. Rev. D 82, 076002 (2010); Moscow Univ. Phys. Bull. 65, 539 (2010).

[11] E.J. Ferrer, V. de la Incera and A. Sanchez, arXiv:1205.4492.

[12] R. Anglani, R. Casalbuoni, M. Ciminale, R. Gatto, N. Ippolito, M. Mannarelli and M. Ruggieri, arXiv:1302.4264 [hep-ph]. 
[13] M. Buballa and S. Carignano, arXiv:1406.1367 [hep-ph].

[14] H. Caldas, J. Stat. Mech. 1110, P10005 (2011). arXiv:1106.0948 [cond-mat.str-el]].

[15] D. Roscher, J. Braun, and J.E. Drut, Phys. Rev. A 89, 063609 (2014).

[16] V. Schon and M. Thies, Phys. Rev. D 62, 096002 (2000); A. Brzoska and M. Thies, Phys. Rev. D 65, 125001 (2002).

[17] O. Schnetz, M. Thies and K. Urlichs, Annals Phys. 314, 425 (2004); C. Boehmer and M. Thies, Phys. Rev. D 80, 125038 (2009); J. Hofmann, Phys. Rev. D 82, 125027 (2010).

[18] G. Basar, G.V. Dunne and M. Thies, Phys. Rev. D 79, 105012 (2009).

[19] D. Ebert, N.V. Gubina, K.G. Klimenko, S.G. Kurbanov, V.C. Zhukovsky, Phys. Rev. D 84, 025004 (2011).

[20] N.V. Gubina, K.G. Klimenko, S.G. Kurbanov and V.C. Zhukovsky, Phys. Rev. D 86, 085011 (2012); Moscow Univ. Phys. Bull. 67, 131 (2012).

[21] A. Chodos, H. Minakata, F. Cooper, A. Singh, and W. Mao, Phys. Rev. D 61, 045011 (2000).

[22] P. Fulde and R.A. Ferrel, Phys. Rev. 135, A550 (1964).

[23] A.A. Andrianov, D. Espriu and X. Planells, Eur. Phys. J. C 73, 2294 (2013).

[24] A.A. Andrianov, D. Espriu and X. Planells, Eur. Phys. J. C 74, 2776 (2014).

[25] R. Gatto and M. Ruggieri, Phys. Rev. D 85, 054013 (2012); M. Ruggieri, arXiv:1110.4907.

[26] L. Yu, H. Liu and M. Huang, arXiv:1404.6969 [hep-ph].

[27] W. Pauli, Nuovo Cimento, 6, 204 (1957); F. Gursey, Nuovo Cimento, 7, 411, (1957).

[28] S. Weinberg, "The quantum Theory of Field II", Cambridge Univ. Press, Cambridge, England, 1996.

[29] K. Fujikawa, Phys. Rev. D 21, 2848 (1980).

[30] K.G. Klimenko, R.N. Zhokhov and V.C. Zhukovsky, Phys. Rev. D 86, 105010 (2012).

[31] M. Thies, Phys. Rev. D 68, 047703 (2003).

[32] I. Ojima and R. Fukuda, Prog. Theor. Phys. 57, 1720 (1977).

[33] A.N. Vasiliev and G.Y. Panasyuk, Theor. Math. Phys. 103, 570 (1995) [Teor. Mat. Fiz. 103, 295 (1995)].

[34] K.G. Klimenko, Theor. Math. Phys. 75, 487 (1988) [Teor. Mat. Fiz. 75, 226 (1988)].

[35] K. Ohwa, Phys. Rev. D 65, 085040 (2002).

[36] D. Ebert, T.G. Khunjua, K.G. Klimenko and V.C. Zhukovsky, Int. J. Mod. Phys. A 29, 1450025 (2014); V.C. Zhukovsky, K.G. Klimenko and T.G. Khunjua, Moscow Univ. Phys. Bull. 68, 105 (2013) [Vestn. Mosk. Univ. Fiz. Astron. 2, 11 (2013)].

[37] A.A. Sokolov, I.M. Ternov, V.Ch. Zhukovsky, and A.V. Borisov, "Kalibrovochnye Polya" ("Gauge Fields") (in Russian), Moscow University Publishing House, Moscow, 1985

[38] L. Jacobs, Phys. Rev. D 10, 3956 (1974); K.G. Klimenko, Theor. Math. Phys. 70, 87 (1987).

[39] A.N. Vasiliev, "Functional methods in quantum field theory and statistical physics", Leningrad Univ. Press, Leningrag, USSR, 1976.

[40] D. Ebert, K.G. Klimenko, Phys. Rev. D80, 125013 (2009).

[41] G. Birkhoff and S. Mac Lane, "A Survey of Modern Algebra", New York: Macmillan, 1977. 